UNIVERSITY

OF WOLLONGONG

AUSTRALIA

\title{
MTKeras: An automated metamorphic testing platform
}

Yelin Liu

This thesis is presented as part of the requirements for the conferral of the degree:

Master of Philosophy

Supervisor:

Associate Professor Zhi Quan (George) Zhou

Co-supervisor:

Professor Willy Susilo

The University of Wollongong

School of Computing and Information Technology

May, 2021 
This work @ copyright by Yelin Liu, 2021. All Rights Reserved.

No part of this work may be reproduced, stored in a retrieval system, transmitted, in any form or by any means, electronic, mechanical, photocopying, recording, or otherwise, without the prior permission of the author or the University of Wollongong.

This research has been conducted with the support of an Australian Government Research Training Program Scholarship. 


\section{Declaration}

I, Yelin Liu, declare that this thesis is submitted in partial fulfilment of the requirements for the conferral of the degree Master of Philosophy, from the University of Wollongong, is wholly my own work unless otherwise referenced or acknowledged. This document has not been submitted for qualifications at any other academic institution.

Yelin Liu

November 15, 2021 


\section{Publications}

Yelin Liu, Yang Liu, Tsong Yueh Chen, and Zhi Quan Zhou, "A testing tool for machine learning applications," in Proceedings of the IEEE/ACM 42nd International Conference on Software Engineering Workshops (ICSEW'20), Seoul, Republic of Korea: ACM, 2020, pp.386-387.

Yelin Liu, Zhi Quan Zhou, Tsong Yueh Chen, Yang Liu and Dave Towey. "MTKeras: An automated metamorphic testing platform" International Journal of Software Engineering and Knowledge Engineering, vol. 31, no. 9, pp.1235-1249, 2021. 


\section{Abstract}

Metamorphic testing (MT) is recognized as an important quality assurance paradigm for complex systems. MT's growing popularity is due not only to its effectiveness in alleviating the test oracle and test case generation problems but also to its unique perspective on software testing. The identification of effective metamorphic relations is vital to metamorphic testing. In addition, a testing platform that allows researchers and testers to automate the process of implementing metamorphic relations can broaden the audience for practical application of metamorphic testing.

By adopting the concept of metamorphic relation patterns, we can effectively implement concrete metamorphic relations. This thesis presents MTKeras, a generic and extensible metamorphic testing platform that implements the concept of metamorphic relation patterns. We demonstrate the platform's applicability and fault-detection effectiveness by conducting case studies on the testing of image classification and sentiment analysis models, search engines, and a database management system. This study not only proves the usefulness of metamorphic relation patterns but also confirms that the composition of metamorphic relations can greatly improve the fault-detection effectiveness of individual metamorphic relations. It also proves that metamorphic relations can help to enhance a user's understanding of the underlying system. We have open-sourced MTKeras. 


\section{Acknowledgments}

I would like to thank my principal supervisor, Associate Professor Zhi Quan Zhou, for his thoughtful support throughout my research process. I would not have been able to finish this research without his guidance. I highly appreciate his patience and knowledge as a supervisor and his critical thinking and academic vision as a scientist. I am also grateful to Professor Willy Susilo for his generous support as my co-supervisor. Thank you also to Professor Dave Towey for helping me with my research. I would also like to thank my parents for supporting me throughout my study period in Australia. Finally, I would like to thank my girlfriend, Bekki, for her companionship during this long journey. 


\section{Contents}

Publications iv

$\begin{array}{lll}\text { Abstract } & \text { v }\end{array}$

1 Introduction 1

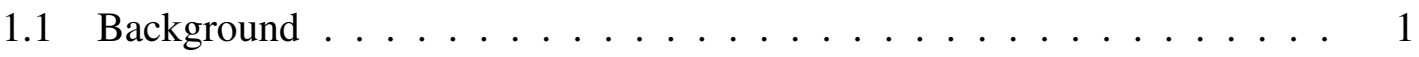

1.2 Motivation and Context . . . . . . . . . . . . . . . 2

1.2 .1 Python ........................ 2

1.2 .2 Keras ......................... 2

1.3 Research Aims . . . . . . . . . . . . . . . . 3

1.4 Contributions ......................... 3

1.5 Structure of the Thesis . . . . . . . . . . . . . . 4

2 Literature Review 5

2.1 Basic Concepts of Metamorphic Testing . . . . . . . . . . . . . . 5

2.2 Systematically Identifying and Implementing MRs . . . . . . . . . 6

2.2.1 Identification and Selection of MRs . . . . . . . . . . . 6

2.2.2 MR Repository . . . . . . . . . . . . . . . . . . . . . 6

2.2.3 Metamorphic Relation Patterns . . . . . . . . . . . . . . 7

2.2.4 Composition of Metamorphic Relations . . . . . . . . . . . . 9

2.3 Implementing MT in Multiple Domains . . . . . . . . . . . . . . . 9

2.3.1 Implementing MT in ML Testing . . . . . . . . . . . . . 9

2.3.2 Implementing MT in Search Engine Testing . . . . . . . . . . . . 11

2.3.3 Implementing MT in Database Management System Testing . . . 12

3 MTKeras: An Automated Metamorphic Testing Platform 13

3.1 Overview . . . . . . . . . . . . . . . . . 13

3.2 Architecture and System Design . . . . . . . . . . . . . . . 13

3.3 Software Features and Functionalities . . . . . . . . . . . . . . . 15

4 MTKeras Case Studies $\quad 18$

4.1 Testing Keras-trained Image-classification Models . . . . . . . . . . . . . 18 
4.1.1 MT using composition/combination of MRs on Image-classification Models . . . . . . . . . . . . . . . . . . 18

4.1.2 Robustness Test on Image-classification Models . . . . . . . . . . 20

4.2 Testing Keras-trained Sentiment Analysis Models . . . . . . . . . . . . . 22

4.2.1 Objectives of the Experiment . . . . . . . . . . . . . 22

4.2 .2 Observation . . . . . . . . . . . . . . . 22

4.2 .3 Evaluation .................... 22

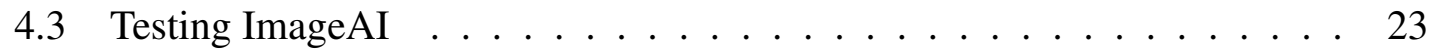

4.3.1 Objectives of the Experiment . . . . . . . . . . 23

4.3 .2 Observation . . . . . . . . . . . . . . 23

4.3 .3 Evaluation ...................... 25

4.4 Testing E-commerce Website Search Engines . . . . . . . . . . . . . . 25

4.4.1 Objectives of the Experiment . . . . . . . . . . . 25

4.4 .2 Observation . . . . . . . . . . . . . 26

4.4 .3 Evaluation ..................... 27

4.5 DBMS Testing on MTKeras . . . . . . . . . . . . . 27

4.5.1 Objectives of the Experiment . . . . . . . . . . . 27

4.5.2 Observation . . . . . . . . . . . . . . . 27

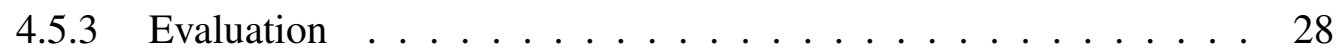

5 Conclusion $\quad 29$

$\begin{array}{ll}\text { Bibliography } & 31\end{array}$

$\begin{array}{lll}\text { A Appendix A } & \mathbf{3 7}\end{array}$

A.1 The results of the ImageAI experiment . . . . . . . . . . . . . . . 37

B Appendix B $\quad 65$

B.1 The execution time of the experiment ............ 65

$\begin{array}{ll}\text { C Appendix C } & \mathbf{7 0}\end{array}$

C.1 Documentation of MTKeras _. . . . . . . . . . . . . 70

C.1.1 MTKeras documentation . . . . . . . . . . . 70

C.1.2 Authors . . . . . . . . . . . . . . . 70

C.1.3 Table of Contents . . . . . . . . . . . . . . 70

C.1.4 Overview ...................... 70

C.1.5 APIs ........................ 71

C.1.6 License ..................... . . 76

C.1.7 Glossary ..................... 76 
D Appendix D $\quad \mathbf{7 8}$

D.1 Survey Design . . . . . . . . . . . . . . . . . 78 


\section{Chapter 1}

\section{Introduction}

\subsection{Background}

Delivering software in good quality is one of the most challenging goals in the software engineering discipline, since contemporary software systems have become highly sophisticated. It is a common practice that more than half of the software development costs are spent on software testing to ensure the software's quality [1]. Therefore, conducting effective software testing can be a main concern of the industry. However, two fundamental problems can challenge the software testing industry, the reliable test set problem and the test oracle problem [2]. The test oracle is a mechanism that can decide whether the output of a test case is correct. If there is an oracle problem, the result of a test case can be difficult or even impossible to verify. In the example of a machine learning application, if it is difficult to tell whether the application is producing the expected output, a test oracle problem is likely to blame.

MT is an effective alleviation for the test oracle problem [3]. It has become recognized as an important quality assurance paradigm for complex systems, such as for machine learning (ML) [4], [5] and database management systems (DBMSs) [6]. MT's growing popularity relates not only to its effectiveness in addressing the test oracle and test case generation problems [7], but is also due to its unique perspective on software testing: Instead of focusing on the correctness of individual outputs of the software under test (SUT), MT examines relations among multiple executions of the SUT. As a result, MT has been able to detect a large number of previously unknown defects in popular ML models [8] and real-life critical systems, such as the onboard LiDAR obstacle perception software in self-driving vehicles [9].

MT has been used to test systems in many diverse domains, including computer vision, machine translation, autonomous systems and cybersecurity [4], [10], [11]. Nevertheless, 
application of MT to ML is still at an early stage, and the identification and coding of metamorphic relations (MRs) generally remains a largely manual process. MRs are the most important components of MT: They are relations among the inputs and outputs of multiple executions of the SUT. An observation that the MRs identified for different application domains often share similarities led to the introduction of metamorphic relation patterns (MRPs) [10], [12]. For example, "equivalence under geometric transformation" is an MRP that can be used to derive concrete MRs for different domains. One concrete MR for the time series analysis domain might be that, when using the Google Cloud Video Intelligence service, the same objects should be detected, regardless of whether the video is played forwards or backwards [10]. Another concrete MR, this time in the domain of autonomous machinery, may be that a drone should not change its behavior in response to transformations (such as rotations) that do not affect its flight route [13].

\subsection{Motivation and Context}

It has been over 20 years since MT was first proposed, and this testing technique has been successfully adopted in many domains. However, promoting MT to researchers and industries is still a significant challenge as the discussion is limited to specific domains. Most of the MT implementations are domain-based, for example, the implementation of applying MT in ML testing [8], [14]-[17] and the application of MT on e-commerce product search engine testing [10], [18]. To expand the audience of the MT community, a generic platform that removes the limitation of domains can be useful. Besides, an effective method for promoting MT is to contribute open-sourced software to communities. To reach a broader audience, we choose Python [19] as our programming language and initially test our platform on the Keras [20] platform.

\subsubsection{Python}

Python [19] is one of the most popular programming languages among researchers. It has a vast library ecosystem to serve multiple purposes that enable researchers to conduct scientific experiments efficiently. Besides, Python is the most frequently used language in ML. Many ML platforms, such as Keras [20], have Python Application Programming Interface (API) for developers.

\subsubsection{Keras}

ML platforms and libraries have become popular, enabling users to develop and train their ML models. Keras is a popular high-level API for neural networks. It works on top of low-level libraries, such as TensorFlow [21], allowing the backend engines to be plugged 
in seamlessly.

Keras makes it possible for users to configure and train a neural network model based on datasets for various tasks, such as image classification or sentiment analysis [20].

\subsection{Research Aims}

This thesis reports on studies conducted to answer the following research questions:

- RQ1: Is it possible to develop a generic, domain-independent, automated MT framework that would allow developers and testers to identify and implement their own MRs?

- RQ2: What would be the applicability and effectiveness of such a framework?

To address RQ1, we developed an automated MT platform, MTKeras, that allows users to define their own MRs based on a prescribed set of operators. We have also conducted preliminary case studies to investigate RQ2.

\subsection{Contributions}

- 1: We have developed an automated metamorphic testing platform, MTKeras, which is open-sourced on Github. ${ }^{\text {a }}$ MTKeras has successfully composited MRPs into concrete MRs and has implemented automatic execution of MT.

- 2: We have conducted empirical studies by using this platform on five applications. The empirical studies illustrate the problem-detection effectiveness of the MTKeras in four domains: image classification, sentiment analysis, search engine and database management system. The applications include models trained on Keras [20], ImageAI [22], the Ebay ${ }^{\mathrm{b}}$ and Tmall ${ }^{\mathrm{c}}$ search engines, and a database management system.

- 3: Xie et al. [23] has collected data from 164 pieces of literature and constructed a website METWIKI ${ }^{\mathrm{d}}$ The author has participated in an extension of the METWIKI project, by contributing to the extraction of MRs from 64 articles and by constructing part of the website.

\footnotetext{
${ }^{a}$ https://github.com/lawrence415610/Mtkeras

b $h$ ttps://www.ebay.com.au/

chttps://www.tmall.com/

${ }^{\mathrm{d}}$ http://www.metwiki.net/
} 


\subsection{Structure of the Thesis}

The thesis is structured as follows: Chapter 2 is the literature review. In Chapter 3, we introduce a generic automated MT platform, MTKeras, which allows users to implement MRPs to generate concrete MRs for MT. Chapter 4 presents five empirical studies in four domains. These five empirical studies demonstrate MTKeras can be used in multiple scenarios, including machine learning models, real-world object detection applications, search engines and database management systems. The final chapter offers a conclusion and future research possibilities related to MTKeras. 


\section{Chapter 2}

\section{Literature Review}

\subsection{Basic Concepts of Metamorphic Testing}

Software testing is challenged by two fundamental problems, the "reliable test set problem" and "test oracle problem" [2]. A test oracle is a mechanism that can verify the test case results. When the test oracle is impossible or difficult to attain, the test oracle problem occurs. The reliable test set problem emerges when a limited number of test cases cannot prove the correctness of the program and it is impossible to conduct exhaustive testing. These two fundamental problems are common in multiple domains, including machine learning testing and database testing.

MT was proposed as a "testing technique to alleviate the test oracle problem" [3]. This technique also enables automated test case generation, providing an effective and lowcost solution to the reliable test set problem. MT is novel because it focuses on relations between the inputs and outputs rather than on the individual execution outputs of the software under test (SUT). It is a useful and simple approach for researchers to adopt, and in recent years it has gained an increasing popularity. When conducting MT, the relations between inputs and outputs are defined as metamorphic relations (MRs), which are the "derived oracles" [10] that can alleviate the test oracle problem. MRs are those properties which are considered essential to the application's functionality. MT focuses on the violation of MRs; If an MR is violated, the program must have some defects.

Typically, MT can be performed with the following four steps: Identify MRs; Define the source test cases, then execute the program and receive the source test case outputs; Generate the follow-up test cases from the source test cases based on MRs, then execute and receive the outputs of the follow-up test cases; Check the source and follow-up test cases' output against MRs. 
For example, take search engine program $\operatorname{Search}(q, c)$, where $q$ and $c$ refer to search criterion and sorting criterion, respectively. The program takes these criteria and keywords as input and returns a list of results as output. However, this search engine program is difficult or impossible to test, as the tester does not know the exact result number that the search engine should return. Thus, we can use MT to alleviate this problem. We can identify an MR: set the same search criterion $q$ for both searches, and the prices are sorted in ascending or descending order as decided by $c 1(c 2)$. These two search inputs should return two outputs with the same results as the output in reverse order. When the results are not equal, the MR is violated. In this case, there are likely defects in the program. In real-world search engine testings, we have identified multiple bugs based on the above MR [10]. Generally, effective MT requires the use of many MRs. However, identification of MRs is still in the preliminary stage, which involves "human intelligence" and "domain knowledge" [23]. Furthermore, little effort is currently devoted to the implementation of MRs in real-world test drivers. Therefore, methods to systematically identify and implement MRs has become a major concern in the MT research community.

\subsection{Systematically Identifying and Implementing MRs}

\subsubsection{Identification and Selection of MRs}

Some studies have developed methodologies for identifying and selecting MRs. Identification of MRs, which is a vital process in MT [24], can be hoc or systematic. Most of the previous studies identify MR in a hoc manner, while some develop methods to systematically identify MRs. One of the methods is called METRIC [25], which provides a "category-choice framework" for automatic MR selection. Additional strategies, such as the "proportional sampling strategy", can also be applied to this problem [26].

Selecting "good" MR that is effective for detecting defects in programs is also important since multiple MRs can be identified in one program [27]. To select "good" MRs, domain knowledge is not enough. There is some research that focuses on how to select proper MRs [27], [28]. However, systematic identification and selection of MRs are still critical challenges in the MT community. One of the most challenging aspects of this problem is automation of the generation process.

\subsubsection{MR Repository}

Before automation of MR generation can be achieved, researchers must collect and reorganize the historical MR data in order to identify patterns. In any case, the studies are still at an early stage. Xie et al. [23] proposed METWIKI, which is a wiki website to store 
and publish MRs that have been found in multiple published works, as they found that the existing MRs can facilitate deduction of new ones. Segura et al. proposed a templatebased approach to describe MRs [29], and the current METWIKI website ${ }^{\text {a }}$ reorganises the existing MR scratches into different domains using such a template. The author has participated in an extension of the METWIKI project, contributing to the extraction of MRs from 64 articles and to construction of part of the website (Fig. 1).

\subsubsection{Metamorphic Relation Patterns}

The dominant MR pattern theory, largely developed over the past twenty years, is summarised by $\mathrm{Wu}$, Sun and Zhou [30], as follows:

"In the early days of MT research, researchers usually identified MRs from scratch for each individual problem under study. To make this process more systematic, Zhou et al. were the first to propose an idea of using an abstract form of MR to derive multiple concrete MRs, and they called this abstract form of MR a "general metamorphic relation" [31]. In a follow-up study, Zhou et al. further identified another type of abstract relation, which is a subset relation among the source and follow-up outputs-they called this a "general relation" [32]. Their empirical results demonstrated that concrete MRs derived from the above abstract forms of MRs had a strong fault-detection capability [31], [32].

Recently, Segura et al. [12] introduced the term metamorphic relation output pattern (MROP), which they defined as an abstract relation among the source and follow-up outputs from which multiple concrete metamorphic relations can be derived. Their work opened a new MT research direction on "metamorphic relation patterns", in a broad sense, as foreseen by Segura in his keynote at the third International Workshop on Metamorphic Testing (ICSE MET'18) [33].

More recently, Zhou et al. [10] further investigated the notion of 'patterns' and formally defined the general concept of a metamorphic relation pattern (MRP) as 'an abstraction that characterizes a set of (possibly infinitely many) metamorphic relations'. Zhou et al. also defined a concept of a metamorphic relation input pattern (MRIP) as 'an abstraction that characterizes the relations among the source and follow-up inputs of a set of (possibly infinitely many) metamorphic relations."”

Similar concepts can also be found in other articles. For example, Murphy [34] proposed seven "classes of metamorphic properties", including "additive", "multiplicate", "permutative", "inversive", "inclusive", "exclusive”, and "compositional”. Murphy cat-

ahttp://www.metwiki.net/ 


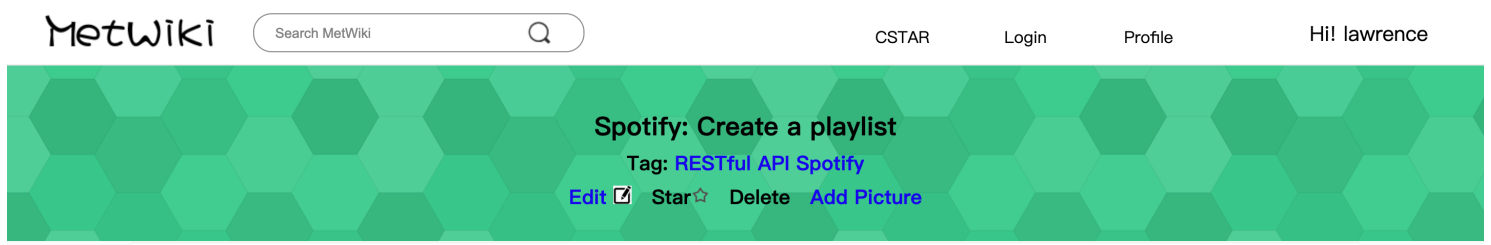

Program Information

Name: Spotify: Create a playlist

Domain: web service and application

Functionality: This service creates a playlist for a Spotify user.

Input:

publicCreat $($ list):; create a public playlist (parameter public=true)

privateCreat (list); ; create a private playlist (public=false)

Output:

$L$ : the created list

Reference

Metamorphic Testing of RESTful Web APIs.

https://dx.doi.org/10.1109/TSE.2017.2764464

MR Information

MR1

Property: Create a playlist. Then, create another playlist with a different name. Both output playlist resources should only differ in the value of the property

name.

Source input: publicCreat(list)

Source output: $L$

Follow-up input:

privateCreat(list)|

Follow-up output: another meme created $L^{\prime} \mid$

Input relation:

only the parameter "public" or "private" changed in both lists

Output relation:

Both output playlist resources should only differ in the value of the property public

Pattern: asymmetry

MR2

Property: Create a playlist. Then, create another playlist with a different name. Both output playlist resources should only differ in the value of the property

name.

Source input: publicCreat(list)

Source output: $L$

Follow-up input:

privateCreat(list)

Follow-up output: another meme created $L^{\prime} \mid$

Input relation:

The playlists are only different in the property name.

Output relation:

Both output playlist resources should only differ in the value of the property name.

Pattern: asymmetry

Figure 2.1: A screenshot for a METWIKI page 
egorised these as "classes of metamorphic properties" since they are "common in mathematical functions". Practically, they can be treated as the MRIPs as they are also the abstract forms of input relation patterns [35].

The concepts of MRP, MRIP and MROP provide a theoretical framework for systematically identifying MRs. Though it is practical to derive solid MRs based on MRP, there has been little implementation of this theoretical framework.

\subsubsection{Composition of Metamorphic Relations}

Instead of deriving MRs using the concept of MRP, compositing existing MRs to derive new MRs has become a popular new technique. Liu et al. [36] proposed this methodology, which they named "composition of metamorphic relations". The main idea is to integrate two or more MRs into one. This methodology is based on the fact that all the MRs must function within the necessary properties of the SUT; When several MRs are composited into one, it should have a higher failure-detection ability than the individual MRs because all the advantages are embedded in the new one.

To sum up, this methodology can improve MT by delivering cost-effective MRs. Efforts toward systematic identification of MRs may help testers effectively derive them and automate the process of MT, which is an important step toward the implementation of MT across domains.

\subsection{Implementing MT in Multiple Domains}

\subsubsection{Implementing MT in ML Testing}

As open-sourced machine learning libraries, such as Keras [20], are widely used, the end-user can effortlessly train their machine learning models by applying those machine learning libraries. However, some machine learning applications can be "safety-critical" [4]. Thus, the quality of those applications must be ensured. Due to the widespread usage of machine learning applications, machine learning testing (ML testing) has received increasing attention in recent years. ML testing is used to describe those activities designed to "reveal the flaws in machine learning items that can result in discordance between the existing and the expected conditions" [4].

The current practice of ML testing is still immature, and there are multiple testing techniques currently being implemented. Among them, MT is one of the most important techniques [4], [5]. As MT has gained popularity, researchers have applied MT to test 
ML systems in multiple domains, such as computer vision, machine translation, and autonomous systems [4], [10]. Murphy et al. [14] was the first research team to apply MT in ML testing. Afterward, an increasing number of studies began to do the same. Xie et al. [37] applied MT in testing supervised machine learning classifiers and found that MRs could represent vital and anticipated properties of the SUT. The violation of these properties reveals defects in the classifiers, and which can utilised for verification. Additionally, violation of the MRs can show the differences between the classifier outcomes and the users' expectation, i.e., it can serve the purpose of validation. Al-Azani et al. [38] also used MT to validate machine learning classifiers, but they applied feature selection techniques along with MT and conducted an empirical study on K Nearest Neighbor (KNN) network.

The robustness of machine learning models can also be tested by MT [4]. This robustness is defined as the "difference" between the correctness of a machine learning system $E(S)$ and the correctness of a perturbated version of this machine learning system $E(\delta(S))$. The robustness can be defined by this equation:

$$
r=E(S)-E(\delta(S))
$$

Arcaini et al. conducted robustness testing on image classification models with MT [39]. They applied MT to test machine learning applications on a specific domain and have revealed many machine learning bugs. As a result, MT has been reported to be able to detect many previously unknown defects in popular machine learning models [8] and in real-life critical systems, such as the onboard LiDAR obstacle perception software of selfdriving vehicles [9].

Other researchers provided testing frameworks or tools for implementing MT in ML testing. For example, Nakajima [15] studied the generalised oracle for ML testing, provided guidelines for MT and presented a coverage criterion for systematically deriving MRs [40]. Ding et al. [16] developed a testing framework for validating the classification accuracy of Convolutional Neural Network (CNN). Tian et al. [8] proposed "DeepTest" for testing Deep Neural Network (DNN) enabled driverless vehicles using MRs. Similarly, Xie et al. [17] proposed "DeepHunter" as a fuzz testing framework for DNN. $\mathrm{Xu}$ et al. [41] utilised MRs to improve classifiers and exposed classification defects. Dwarakanath et al. [42] applied MT to reveal machine learning bugs in the implementation of image classifiers.

Nevertheless, the current practice of implementing MT to ML is still immature. Particularly, the identification of MRs is still mainly a manual process, as is the implementation 
of MRs into test drivers. Most prior implementations of MRs have been targeted toward a specific domain. However, it has been observed that MRs identified within different application domains often share similar viewpoints; Hence, there is a need for introduction of the concept of MRPs into ML testing [10], [12]. For example, "equivalence under geometric transformation" is an MRP that can be used to derive a concrete MR for the time series analysis domain and another concrete MR for the autonomous driving domain [10]. Thus, it is possible for us to propose a generic testing tool across multiple domains of ML testing, and it follows that MT can be effectively applied to test machine learning applications. However, the amount of prior research that concerns the automation of MT is limited. More studies (studies like [8] and [17]) and further empirical studies are needed to practically apply MT in ML testing. Similarly, only a few articles have been written on neural networks, such as RNN and CNN, and more work is needed in this field.

\subsubsection{Implementing MT in Search Engine Testing}

MT was introduced to automate search engine testing by Zhou et.al [31]. The "functional correctness" of the web search engines can scarcely be tested due to the test oracle problem [31]. To be more specific, it is difficult for testers to tell whether the results returned by a search engine are complete and correct. Even if a test oracle exists, most of the approaches to search engine testing depend on "relevance judgments" of the search outputs [43]. Thus, testing a search engine can be laborious and prone to error. MT can alleviate the test oracle problem and facilitate test case generation. Although identity relations are widely employed in software testing, the methodology of MT is unique in that it allows for application of "non-identity" relations [31]. This makes MT a very useful technique for search engine testing.

In web search engine testing, the relations between search queries, keywords and results can be used as test oracles, and the process of mining these test oracles can be automated. Zheng et al. [44] implemented their approach on multiple search engines and demonstrated that implementation can facilitate mining "high-confidence rules". Other research focused on e-commerce websites' search engines, which can be different from web search engines like Google and Yahoo [18]. Due to the presence of MR, the MT methodology can be used to test any of these search engines, regardless of the backend mechanics. As MRP is conceptually applied in automating MT, it can also be effective in testing search engines. From an end user's perspective, this methodology improves understanding of the search engine system [10]. 


\subsubsection{Implementing MT in Database Management System Testing}

As MT can be used to test "non-testable" systems, it is also implemented in Database Management System (DBMS) testing. A DBMS is a software package designed to allow easy manipulation of a database [45]. DBMS is essential in many systems but difficult to test. A significant obstacle to improve database performance is upgrading the search query, which is complicated. Testing the query optimizer has become the most challenging task associated with testing a DBMS [46]. There are multiple methods for detecting bugs in a DBMS, such as Pivoted Query Synthesis (PQS) [47] and Random Generation of SQL (RAGS) [48]. However, the major drawback of these techniques is that they are only effective on the "common core" of Structured Query Language (SQL), and only few research is conducted on this issue. Researchers developed a novel method called NonOptimizing Reference Engine Construction (NoREC) [6], which compares the results of the optimized and non-optimized versions of a SQL to evaluate the DBMS. The key insight of this research is that the SQL can always be rewritten to a different SQL but has the same functionality. This process is automatically done by the DBMS. In the study, some SQL is rewritten by the researcher to mimic the automatic process of the DBMS. Interestingly, the methodology of NoREC can be taken as an MR if the testing process is considered to be an MT. It is simple and effective to compare the results of the two test versions of the SQL due to the effectiveness of MR. Database management system testing is also challenged by the test oracle problem [6]. Since a database management system provides little control over its optimizations, it can be difficult to obtain the test oracle. However, the concept of MRP can also be applied to automate the process of MT for database management system testing. 


\section{Chapter 3}

\section{MTKeras: An Automated Metamorphic Testing Platform}

\subsection{Overview}

MTKeras is designed with usability and reusability in mind, for researchers and practitioners who do not have much prior knowledge of MT to implement it. Thus, the user interface needs to be simple and the design should be extendable. Further, the tool should support a popular programming language and a variety of application domains. We have developed MTKeras by using Python [19], and first tested it on Keras [20]

MTKeras enables automated MT by providing users with an MR library that is based on a hierarchical structure (with levels of abstractions) of MRPs [10]. MTKeras also allows users to define and run new MRs through composition of multiple other MRs [36], [49]. Given source test cases provided by the users, MTKeras generates follow-up test cases according to the relevant $\mathrm{MR}(\mathrm{s})$, automatically recording any violations and return test results.

\subsection{Architecture and System Design}

MTKeras was designed around two basic concepts: metamorphic relation input patterns (MRIPs) [10]; and metamorphic relation output patterns (MROPs) [12]. MRIPs and MROPs describe relations between the source and follow-up inputs and outputs, respectively (Fig.3.1). Both MRIPs and MROPs can have multiple levels of abstractions. Some example MRIPs include: replace, which involves changing the value of part of the input (such as in Sun \& Zhou's $M R_{\text {replace }}$ [50]); noise, which adds noise-a dot in an image, for example - to the input data (as done by Wu et al. [30]); and additive and multiplicative, 

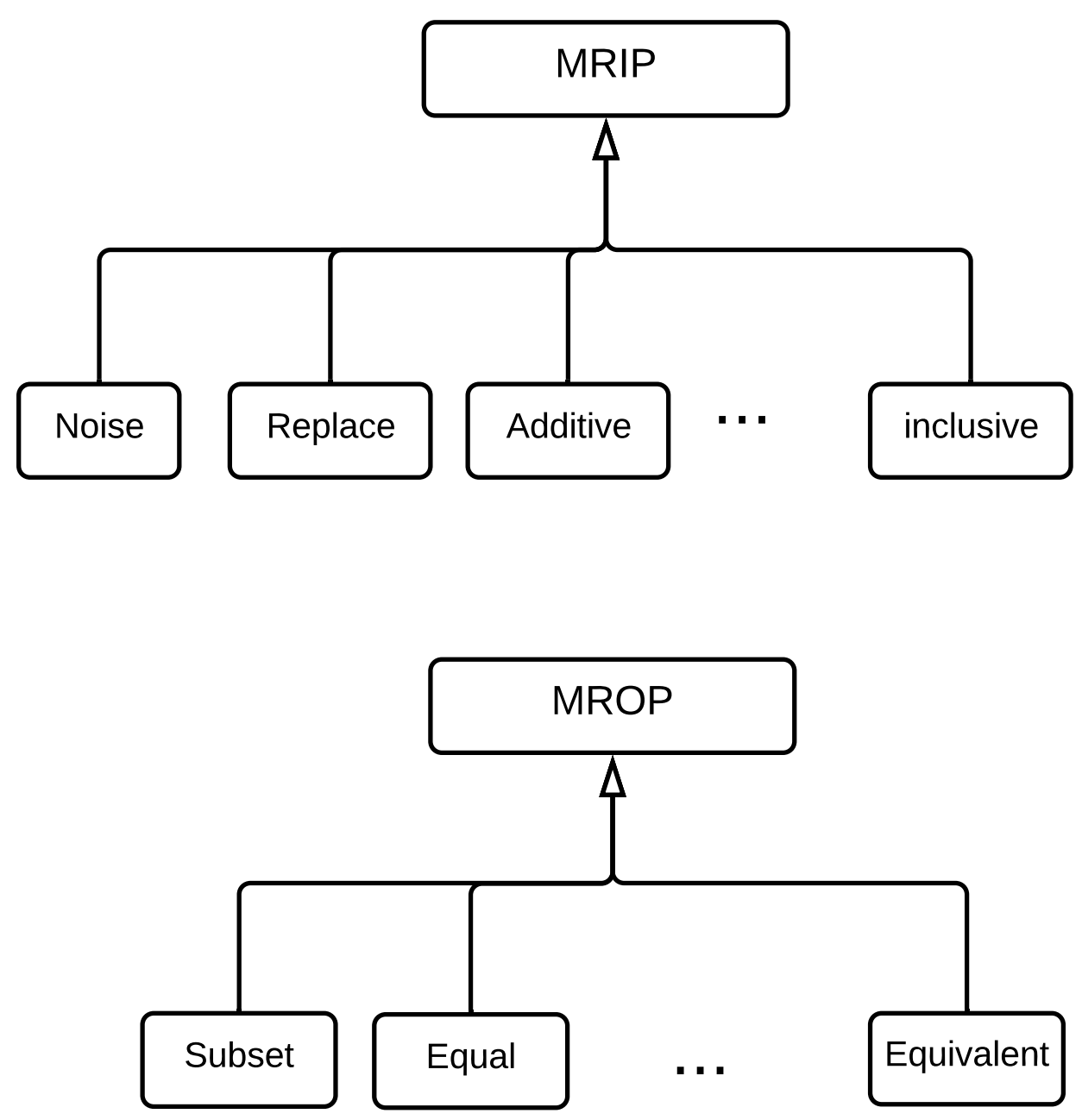

Figure 3.1: MTKeras Concept 


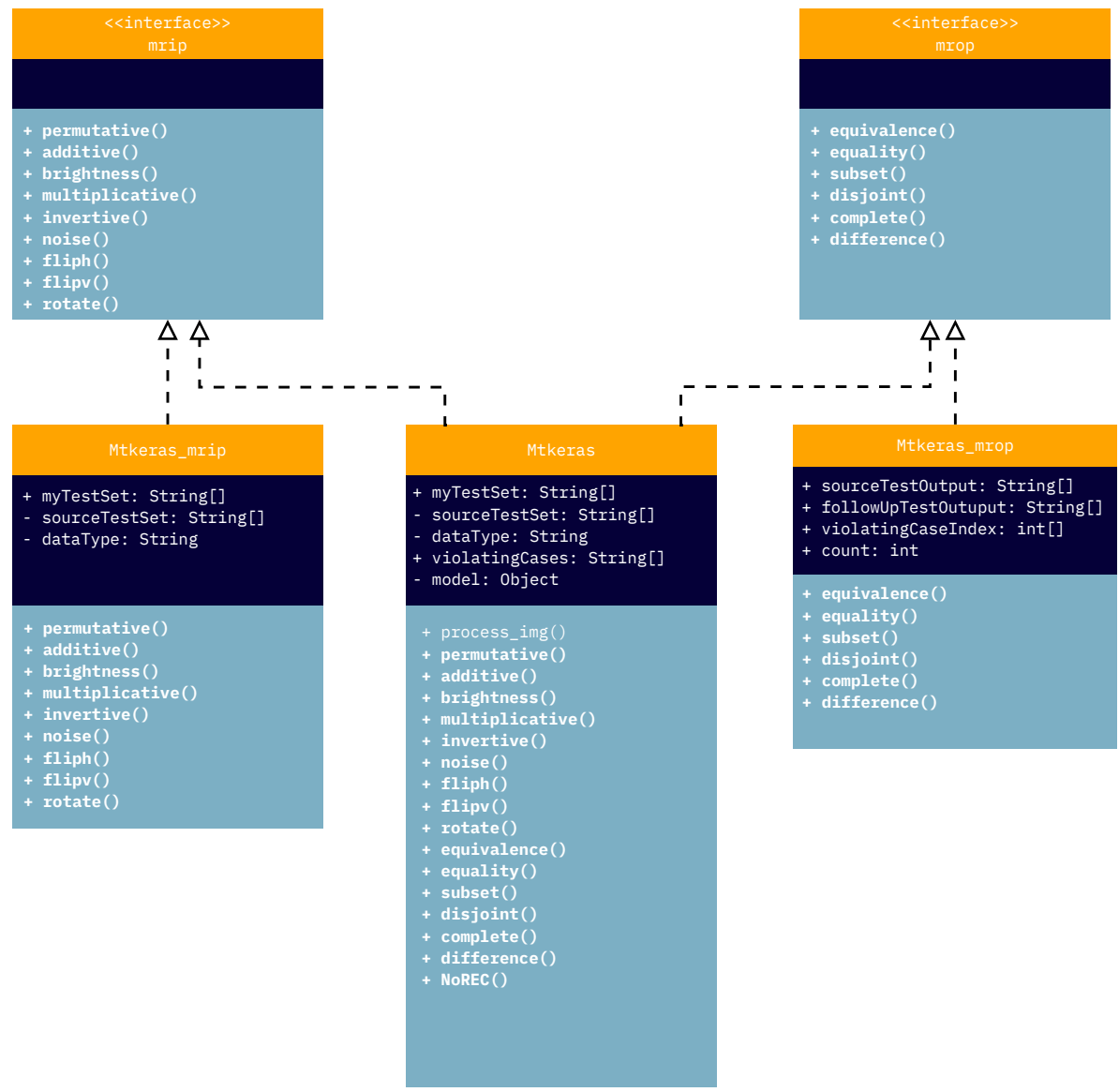

Figure 3.2: MTKeras class diagram

where the input is modified through addition or multiplication, respectively (as seen in the "metamorphic properties" defined by Murphy et al. [34]). Examples of MROPs include: subsume/subset, where the follow-up output should be a superset/subset of the source output [32]; equivalence, where the follow-up and source outputs should be equivalent [12]; and equal, where the follow-up and source outputs should contain the same items, in the same order [12].

MTKeras provides three APIs, including the "Mtkeras", "Mtkeras_mrip" and "Mtkeras_mrop". In MT, each call of the APIs generates a test case that can perform the functionalities mentioned above. A class diagram (Fig.3.2) of the system design is illustrated.

\subsection{Software Features and Functionalities}

MTKeras is a fully-automated platform that facilitates MT for users (both industrial and academic) who may not have much experience in metamorphic testing. MTKeras is ex- 
tensible: As more MRIPs and MROPs are identified and coded, they can be used to generate MRs that are configured by end-users. Through application of MRIP combination, derived MRs can be combined to create new MRs, which themselves can be further combined to create even more new MRs, and so on, thus providing numerous effective MRs for MT. For ease of use, MTKeras has been implemented as a python package that can be downloaded from Github ${ }^{\mathrm{a}}$ and imported as a Python module. Once imported, users can easily choose MRIPs and MROPs, and configure concrete MRs writing a single command in the following format:

$$
\operatorname{Mtkeras}(<\text { sourceTestSet }>,<\text { dataType }>\text { ). }<\text { MRIPs }>[.<\text { MROP }>]
$$

where $<$ sourceTestSet $>$ specifies where the source test cases are stored; $<$ dataType $>$ declares the type of each element (test case) of $<$ sourceTestData $>$ (e.g., grayscaleImage, colorImage, searchTerm, etc.); <MRIPs $>$ represents a sequence of one or more MRIPs; and $<$ MROP $>$ represents an optional MROP. Although multiple MRIPs are possible in a single configured MR, only one MROP can be used. For example, a command to test an image classification model could be:

\section{Mtkeras(myTestSet, colorImage).noise(1).fliph().equal()}

which tells MTKeras to use the array "myTestSet" as the set of source test cases, where each test case is a color image; follow-up test cases are to be generated by adding a noise point to each image and then horizontally flipping it. The last term, ".equal()", tells MTKeras to check whether or not the classification results for the source and follow-up test cases are the same. Note that the MROP ".equal()" is optional: If omitted, MTKeras will return a set of follow-up test cases without further testing. This set of follow-up test cases can be used for MT or for any other purpose, such as data augmentation [24]. The MROP and MRIP interfaces can also be used independently. The following command generates a data set of color images, each of which has been flipped horizontally and contains a noise point:

Mtkeras_mrip(myTestSet, colorImage).noise(1).fliph()

The following command instructs MTKeras to check whether or not the source and follow-up outputs violate the MROP "equal":

$$
\text { Mtkeras_mrop(sourceOutput, followupOutput).equal() }
$$

MTKeras can be automated to perform MT and report all the violating cases. We provide

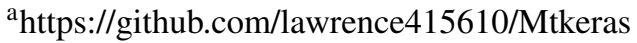


the documentation of the MTKeras (see Appendix C). 


\section{Chapter 4}

\section{MTKeras Case Studies}

MTKeras is a testing framework based on the concept of MRPs. The nature of MRPs means that MTKeras can be used in various domains, regardless of whether or not the SUT is an ML model. This section reports on a series of case studies involving application of MTKeras in five different domains. There are two main reasons to choose these five domains: they are popular, and their SUTs center around data. We first report on the testing of ML models trained on the Keras platform: an image-classification model trained with the MNIST dataset (Section 4.1), and a sentiment-analysis model trained with the IMDB dataset (Section 4.2). We then examine MTKeras applied to ImageAI, a real-life object-detection application (Section 4.3). Finally, we discuss the use of MTKeras for search-engine testing (Section 4.4) and for database testing (Section 4.5).

Among the SUTs, the models trained on the Keras platform and the ImageAI application are machine learning applications, while the search engine and DBMS are conventional data-processing applications. The case studies demonstrate the effectiveness of MRPs and the use of combined MRs.

\subsection{Testing Keras-trained Image-classification Models}

\subsubsection{MT using composition/combination of MRs on Image-classification Models}

\section{Objectives of the Experiment}

In this experiment, we would like to show that MTKeras can painlessly achieve the composition/combination of MRs. We test our platform on top of Keras since Keras can provide multiple ML models for research. Our first experiment tests widely-used image classification models. If MTKeras can support composition/combination of MRs on these testing image classification models, it can be used to communicate the concept of MRP 

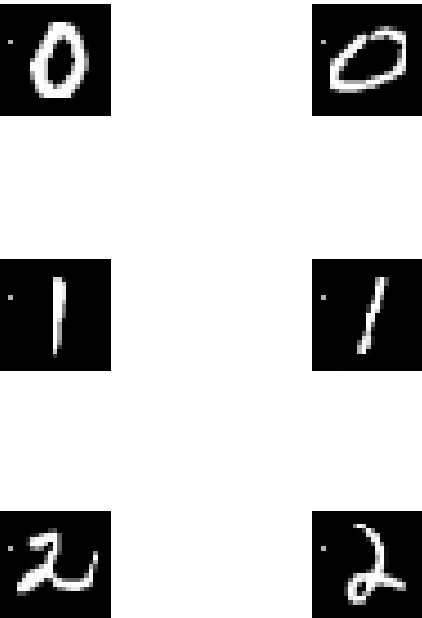
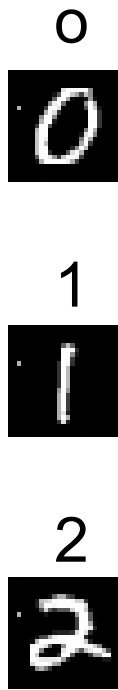
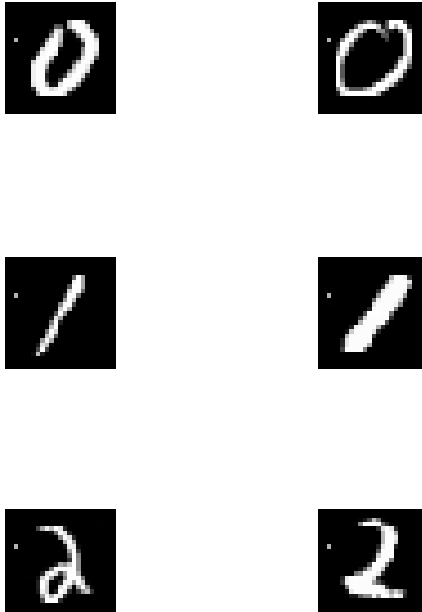

Figure 4.1: Adding one pixel of noise to the dataset

to a broad audience.

\section{Observation}

The ML models tested were taken from the official Keras website [20]. Our first study involved the MNIST handwritten digit dataset [51], which contains 70,000 28*28-pixel grayscale images of handwritten digits. 60,000 and 10,000 images were used as the training and test sets, respectively. The Keras model has a $98.40 \%$ testing accuracy. We first conducted an MRIP combination experiment on this model.

To test this ML model, we defined the following two MRs:

- MR $R_{\text {brightness: }}$ : Reducing the brightness (by 10\%) of each of the images should not improve the ML model's accuracy because, intuitively, a darker image should be more difficult to recognize.

- $M R_{\text {noise }}$ : Adding a random noise point to each image should not improve the ML model's accuracy because, intuitively, an image with an extra random point should be more difficult to recognize (Fig. 4.1).

As expected, our experiments with $M R_{\text {brightness }}$ identified zero violations. However, out of 1000 experiments (thus 1000 pairs of source and follow-up accuracy scores), $M R_{\text {noise }}$ was violated by 387 , meaning that in $38.7 \%$ of the situations, adding a random noise point increased the model's accuracy. A more interesting observation was that the combination of $M R_{\text {brightness }}$ and $M R_{\text {noise }}$ yielded a violation rate of $45.5 \%$ (455 out of 1000). 


\section{Evaluation}

In this experiment, we successfully implement the composition/combination of MRs feature into our test drivers. By applying MTKeras, we can batch process the MT test cases which also provides further evidence that composition/combination of MRs can increase the fault-detection effectiveness [52].

\subsubsection{Robustness Test on Image-classification Models}

\section{Objectives of the Experiment}

We next conduct a robustness test of image-classification models - this relates to a classifier's ability to classify similar inputs into the same category [39]. A frequently-used robustness test involves the generation of adversarial examples. For example, when testing a Convolutional Neural Network $(\mathrm{CNN})$ - a class of deep neural network commonly used for image classification - we can generate some transformed data by rotating the source input [39]. This is supported by MTKeras, which uses MRIPs to generate transformed images. By conducting this experiment, we show that MTKeras can be applied for multiple purposes, such as robustness test.

\section{Observation}

We used the CIFAR-10 dataset - which contains 60,000 32*32 color images in ten different classes - to train another image classification model (using Keras) for comparison with the MNIST model. This allowed us to compare image-classification models trained using grayscale images with models trained using color images. The "noise" and "rotate" MRIPs were used to test the MNIST and CIFAR-10 models. MTKeras can be used to easily implement MRIPs, and to record the accuracy of the relevant model under test. The following command, for example, will generate a dataset with images that have all been rotated by 5 degrees:

Mtkeras_mrip(myTestSet, colorImage).rotate(5)

80 rounds of testing were conducted. Tables 4.1 and 4.2 present the experiment results.

\section{Evaluation}

In this experiment, we show that MTKeras can effortlessly support robustness tests on image classification models. Besides, there is a non-monotonic decreasing trend in both models' accuracy when we incrementally add noise points and increase the degree of rotation. 


\begin{tabular}{|l|l|l|}
\hline Noise (1*1 pixel points) & MNIST accuracy & Cifar10 accuracy \\
\hline 0 & 0.9909 & 0.7415 \\
\hline 40 & 0.9885 & 0.6551 \\
\hline 80 & 0.9854 & 0.5551 \\
\hline 120 & 0.9808 & 0.5266 \\
\hline 160 & 0.9734 & 0.3452 \\
\hline 200 & 0.9549 & 0.3911 \\
\hline 240 & 0.9147 & 0.2634 \\
\hline 280 & 0.9253 & 0.2798 \\
\hline 320 & 0.9559 & 0.2504 \\
\hline 360 & 0.8438 & 0.2252 \\
\hline 400 & 0.7574 & 0.2225 \\
\hline 440 & 0.9015 & 0.2247 \\
\hline 480 & 0.9006 & 0.2042 \\
\hline 520 & 0.8172 & 0.2229 \\
\hline 560 & 0.8553 & 0.1643 \\
\hline 600 & 0.8172 & 0.1880 \\
\hline 640 & 0.8168 & 0.2139 \\
\hline 680 & 0.7001 & 0.2168 \\
\hline 720 & 0.7940 & 0.1798 \\
\hline 760 & 0.7603 & 0.1460 \\
\hline & & \\
\hline
\end{tabular}

Table 4.1: Noise robustness testing of two image classification models.

\begin{tabular}{|l|l|l|}
\hline Rotation (degrees) & MINST accuracy & Cifar10 accuracy \\
\hline 0 & 0.9909 & 0.7415 \\
\hline 1 & 0.9906 & 0.7463 \\
\hline 2 & 0.9899 & 0.7282 \\
\hline 3 & 0.9866 & 0.6822 \\
\hline 4 & 0.9807 & 0.6155 \\
\hline 5 & 0.9662 & 0.5200 \\
\hline 6 & 0.9316 & 0.3987 \\
\hline 7 & 0.8617 & 0.2899 \\
\hline 8 & 0.7317 & 0.2153 \\
\hline 9 & 0.5556 & 0.1728 \\
\hline 10 & 0.3722 & 0.1531 \\
\hline 11 & 0.2357 & 0.1404 \\
\hline 12 & 0.1558 & 0.1338 \\
\hline 13 & 0.1274 & 0.1328 \\
\hline 14 & 0.1394 & 0.1302 \\
\hline 15 & 0.1910 & 0.1294 \\
\hline 16 & 0.2369 & 0.1314 \\
\hline 17 & 0.2616 & 0.1341 \\
\hline 18 & 0.2601 & 0.1293 \\
\hline 19 & 0.2472 & 0.1298 \\
\hline
\end{tabular}

Table 4.2: Rotation robustness testing of two image classification models 


\subsection{Testing Keras-trained Sentiment Analysis Models}

\subsubsection{Objectives of the Experiment}

Sentiment analysis is another popular domain. By testing sentiment analysis models trained on Keras, we would like to show that MTKeras is able to conduct MTs across different domains. Besides, we aim to demonstrate that MRs can help to enhance the user's understanding of the system.

\subsubsection{Observation}

We used MTKeras to test four different ML models, each trained on the same IMDB sentiment-classification dataset, the reviews in which were labeled " 1 " for positive and "0" for negative feelings. The four ML models under test were: CNN (a convolutional neural network based on "shared-weights" and "translation invariance characteristics" [53]); RCNN (Recurrent CNN - a CNN that uses a recurrent structure [54]); FastText (a framework for quickly training text-classification models [55]); and LSTM ("long short term memory", which includes forgetting in the ML model-training [56]). The following MR, related to the permutative MRIP [34], was used in the testing:

- $M R_{\text {permute }}$ : Randomly shuffling (permuting) the words in each movie review shall dramatically reduce the accuracy of the ML models.

The validity of $M R_{\text {permute }}$ is obvious, as shuffling the words should make the sentences meaningless. For example, an original movie review excerpt ("don't you think the whole story was so lovely because it was true") would become, after permutation, a modified (follow-up) test case (“don't was the true whole so lovely think story it you was because”).

In spite of the intuitive and obvious validity of $M R_{\text {permute }}$, the experimental results were surprising. Fig. 4.2 shows the results from 100 MT rounds: Shuffling the words only decreased the accuracy by a very small amount (less than $8 \%$ ), indicating that the ML models under test were insensitive to word order. Furthermore, the accuracy of the FastText model did not change at all, potentially indicating a defect.

\subsubsection{Evaluation}

The experiment demonstrates that MTKeras can be effortlessly applied to different domains. The MRs in sentiment analysis can be vastly different from image classification. However, MRPs, such as "permute" in this experiment, can be applied regardless of domains. This case study also shows that MRs can help to enhance system understanding, confirming our previous report [10]. 


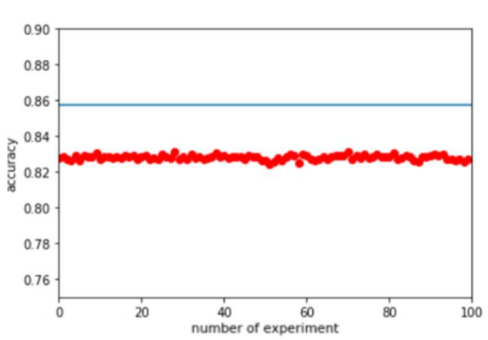

RCNN

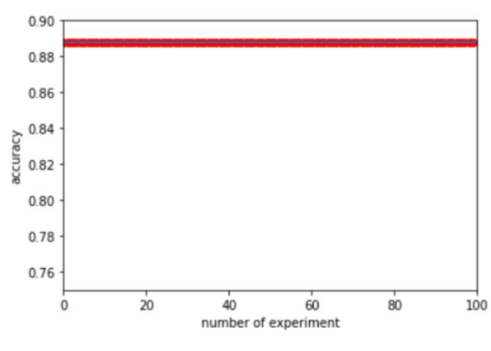

FastText

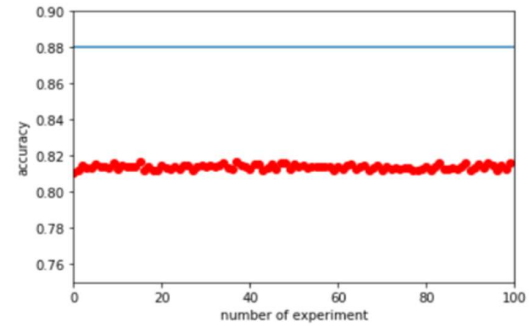

CNN

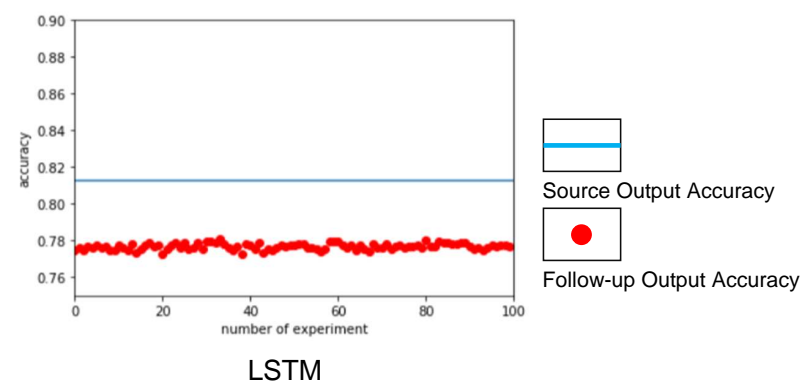

Figure 4.2: Accuracy changes for four neural networks for $M R_{\text {permute }}$

\subsection{Testing ImageAI}

\subsubsection{Objectives of the Experiment}

The previous experiments are conducted on ML models trained on Keras mainly for research. MTKeras has more potential to be applied on production-level machine learning applications. We aim to test how efficient MTKeras could be, to be applied to such applications. Besides, we would like to compare multiple experiments and reveal some patterns of the combination/composition of MRs.

\subsubsection{Observation}

We conducted MRIP combination experiments on the real-life object-detection application ImageAI. ImageAI is an open-source ML application that enables developers and researchers to perform object detection in real-life scenarios [22].

The experiments revealed a number of regular patterns for MRIP combination. ImageAI provides three deep learning models for object detection, all of which offer the same functionality, but differ in prediction time and accuracy: RetinaNet has high performance and accuracy, but longer detection time; YOLOv3 has moderate performance, accuracy and detection time; and TinyYOLOv3 has moderate performance and accuracy with fast detection time [22].

We tested the three models with the open source ApolloScape Open Dataset [57] from 


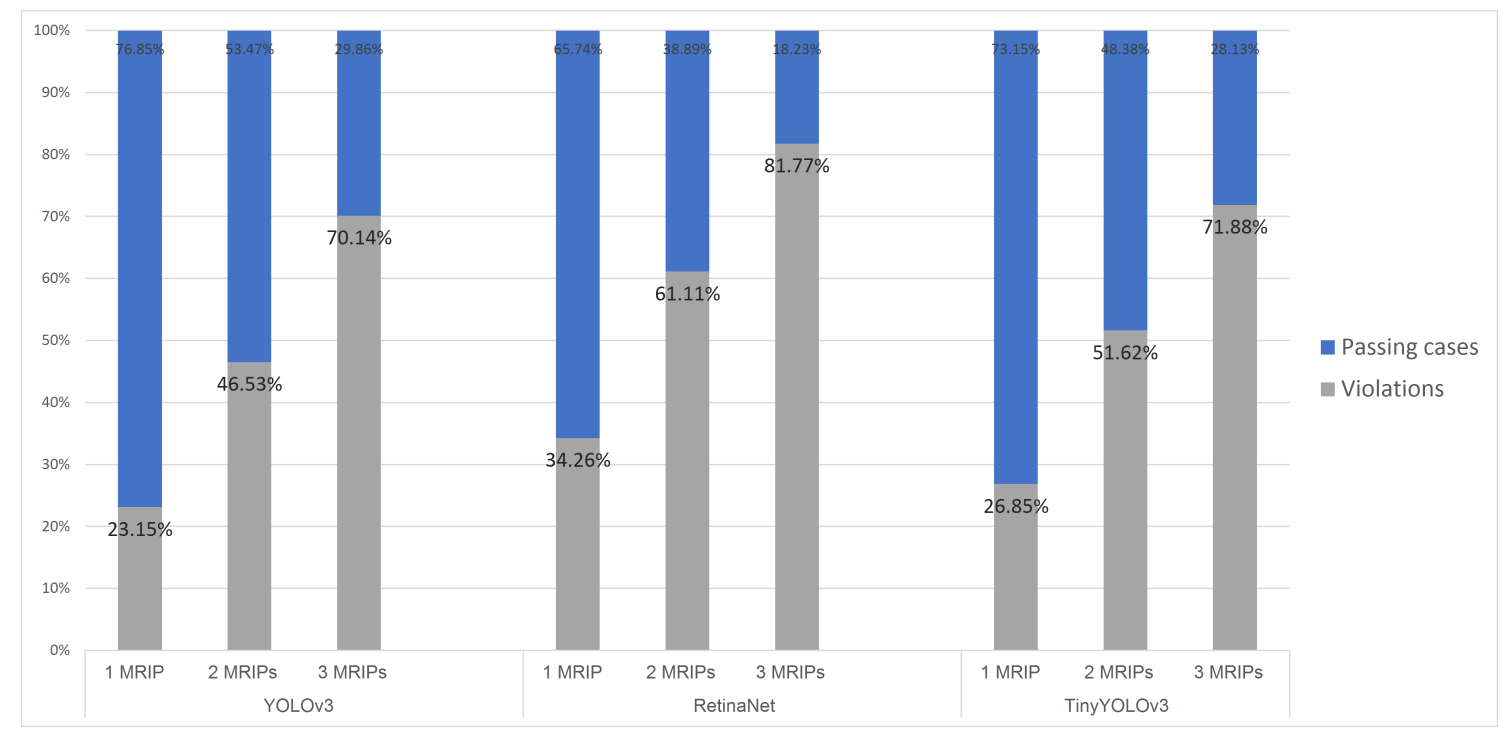

Figure 4.3: ImageAI MRIP combination experiment results

Baidu Apollo, which consists of a set of pictures $(3384 * 2718$ pixel) captured by dashboard cameras. ImageAI was used to detect the number of cars in the ApolloScape pictures. The MRIPs applied in this experiment were "noise", "brightness" and "rotate"; and the MROP was "equal". Multiple more-concrete MRIPs can be generated from each of these MRIPs - four more can be generated from the "noise" MRIP, for example, by adding 10,100,1000, and 10,000 1*1 pixel noise points. MTKeras makes it easy to configure a batch of MRs by combining the MRIPs and MROPs. For example, the following command can be used to configure an MR, meaning that adding ten $1 * 1$ pixel noise points and rotating by five degrees should not change the number of cars detected by the application:

\section{Mtkeras(myTestSet, colorImage).noise(10).rotate(5).equal()}

MTKeras returns whether or not the MR is violated by comparing the number of cars detected in both the original and altered images. Fig. 4.3 summarizes the experimental results (also see Appendix A), which were based on different data for each model.

The YOLOv3 results show that, when the MR represented only one MRIP, the violation rate was $23.15 \%$ (25 out of 108); when representing two MRIPs, the violation rate was $46.53 \%$ (201 out of 432); and when the MR represented three MRIPs, the violation rate was $70.14 \%$ (404 out of 576). The RetinaNet results show that, when the MR represented only one MRIP, the violation rate was $34.26 \%$ (37 out of 108); when representing two MRIPs, the violation rate was $61.11 \%$ (264 out of 432); and when the MR represented three MRIPs, the violation rate was $81.77 \%$ (471 out of 576). The TinyYOLOv3 results show that, when the MR represented only one MRIP, the violation rate was $26.85 \%$ (29 
out of 108); when representing two MRIPs, the violation rate was 51.62\% (223 out of 432); and when the MR represented three MRIPs, the violation rate was $71.88 \%$ (414 out of 576).

Execution time in the experiment consisted of two parts, execution time of the ImageAI application, and the MTKeras execution time. Although applying different models will not change the MTKeras execution time, the number and type of MRIPs combined in each MR does have an impact (Appendix B). The average execution time for an MR involving one "noise" MRIP was 0.66 seconds; for a "brightness" MRIP, it was 1.06 seconds; and for a "rotate" MRIP, it was 14.54 seconds.

For MRs involving two or more MRIPs, the execution time was approximately the sum of each MR with one MRIP combined: The average execution time for the MR combining "rotate" and "brightness" was 14.81 seconds; for the combination of "rotate" and "noise", it was 14.98 seconds; and for the combination of "brightness" and "noise", it was 1.63 seconds. The average execution time for the MR combining all three MRIPs was 17.19 seconds. As expected, increasing the number of MRIPs resulted in requiring more execution time. Regardless of the model and the data used, the composition experiments with Image AI reveal a regular pattern: Increasing the number of MRIPs used to build the MR monotonically increased the violation rate.

\subsubsection{Evaluation}

MTKeras is simple and highly efficient in the scenario of conducting MT on a real-life machine learning application. Through the experiments, we show that MTKeras has the potential to be applied on the production-level automatic testing. Besides, we reveal a regular pattern that can help us understand the effect of the composition/combination of MRs.

\subsection{Testing E-commerce Website Search Engines}

\subsubsection{Objectives of the Experiment}

Although MTKeras is designed as a tool to test ML models, we extend its usage to other domains, including search-engine testing and database testing. This versatility and extensibility are due to MTKeras's use of MRPs, which are naturally generic and can be applied across domains. This characteristic of MTKeras is significant: It removes limitations of previous research [10], [12] and extends MTKeras' applicability to different domains. In this experiment, we demonstrate that MTKeras can be applied to e-commerce website 


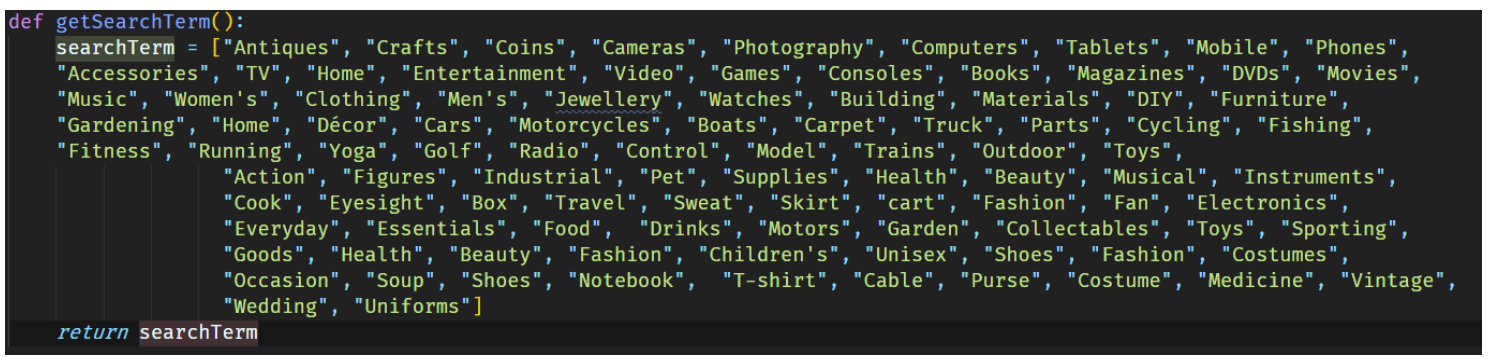

Figure 4.4: A screenshot of the 100 search terms used when testing the e-commerce search engines

search engine testing.

\subsubsection{Observation}

Our next experiment used MTKeras as an MT tool to test the stability of the search engines used on the e-commerce websites Tmall ${ }^{\mathrm{a}}$ and Ebay. ${ }^{\mathrm{b}}$

Both Tmall and Ebay use search engines to allow users to search for various goods. To test the stability, the same experiment was repeated at different times to see whether or not the search engines returned the same results set.

The experiment involved entering 100 search terms (Fig. 4.4) into the search engine, one term at a time, as the source inputs, and then repeating the same searches one minute later, five minutes later, and 30 minutes later. The results from each repeated search were checked and compared. Ideally, the search engine should return the same (or similar) results for each query — some small modifications made during the period may result in slightly different results. The search engine should not return a result that has a comparatively large difference, as this would indicate the presence of a defect in terms of stability. The following MR was used for this testing:

- $M R_{\text {time }}$ : When repeating a search using a particular keyword on a search engine, the search results returned should not change dramatically over a short period of time.

This MR was specified using the following command:

Mtkeras(mySearchTerm, searchTerm).equal(params)

The experimental results showed that the search engines could indeed demonstrate unexpected changes. For example, when "Unisex" was input as a search term, Ebay returned 2,806,666 items, but just one minute later, the result changed to 3,175,969 items: Within

\footnotetext{
ahttps://www.tmall.com/

bhttps://www.ebay.com/
} 
the space of one minute, the same search term could return a result with 369,303 more items than the previous search.

Using MTKeras, we found that the violation rate of $M R_{\text {time }}$ increased as the interval between searches increased. For the Ebay search engine, with a one-minute interval, the violation rate was $34 \%$; for five minutes, the violation rate was $93 \%$; and for 30 minutes, the violation rate was $94 \%$. For the Tmall search engine, for a one-minute interval, the violation rate was $21 \%$; for five minutes, the violation rate was $83 \%$; and for 30 minutes, the violation rate was $84 \%$. From a user's perspective, this amount of changeability and uncertainly in behavior may lead to confusion.

\subsubsection{Evaluation}

The experiment reveals issues with both websites' search engines and again shows how MRs can help enhance system understanding [10]. We show that MTKeras can be applied to search engine testing and the MR of the search engines' stability test can be helpful for further research.

\subsection{DBMS Testing on MTKeras}

\subsubsection{Objectives of the Experiment}

As we mentioned in the previous section, MTKeras removes the limitation of domains, and we extend the application of MTKeras to DBMS testing. Through this experiment, we aim to demonstrate that MTKeras is able to be applied on testing production-level applications based on domain-specific MRs.

\subsubsection{Observation}

MTKeras was also configured for database management system (DBMS) testing by using the concept of Non-Optimizing Reference Engine Construction (NoREC) [6]. Comparing the results of optimized with non-optimized SQL queries can be done using the following MR:

- $M R_{\text {SameDBMS }}$ : When an SQL query is rewritten as an optimized version, the number of results returned by the DBMS should remain the same.

This MR can be specified in MTKeras with the following command:

$$
\text { Mtkeras(mySQL, SQL).NoREC().equal() }
$$




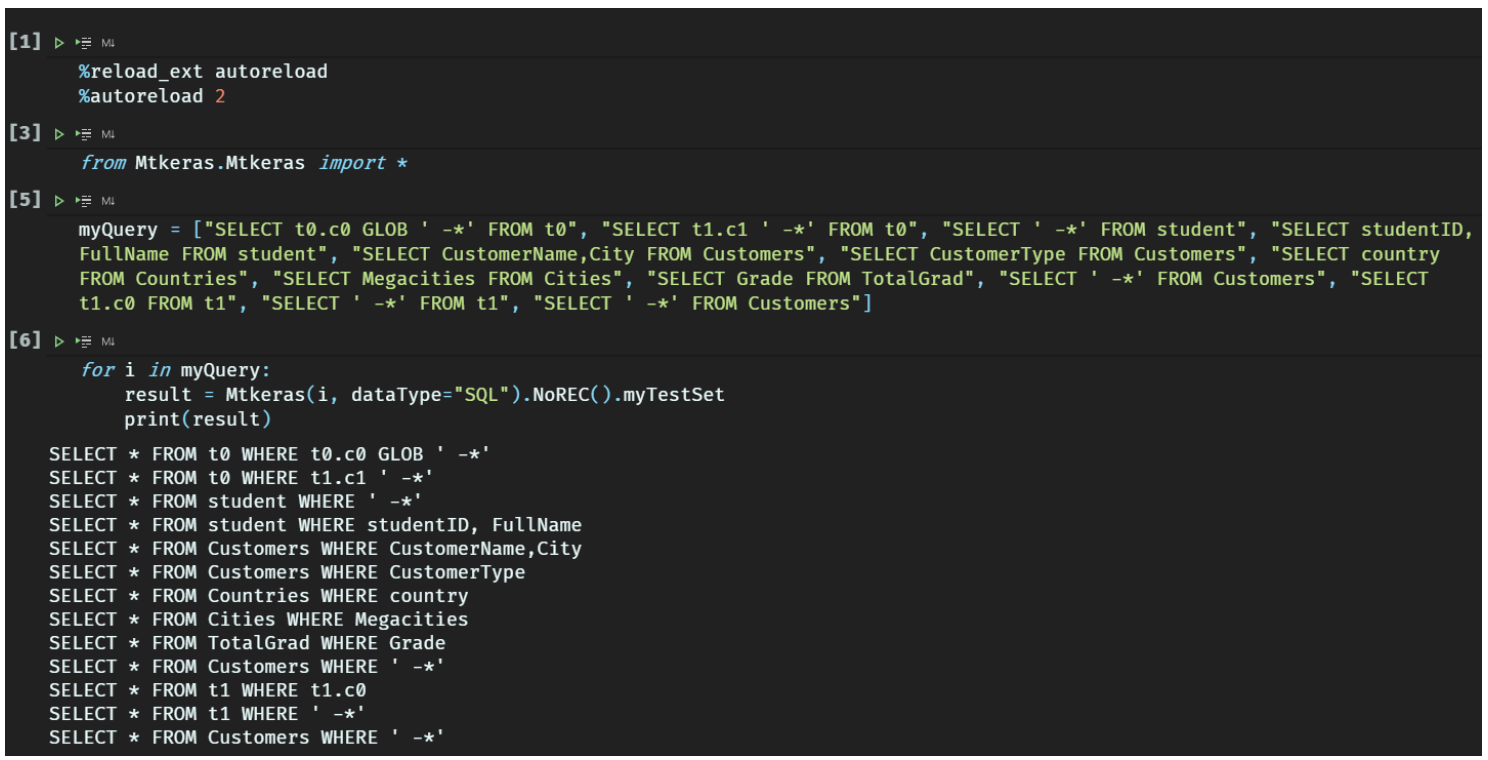

Figure 4.5: A screenshot of MTKeras generating SQL queries for MT

In this case, the NoREC method is taken as an MRIP, and the source input is rewritten to become the follow-up input. Due to the effectiveness of the MRP, DBMS testing could be conducted without needing to know many details about the NoREC methodology (Fig. 4.5).

\subsubsection{Evaluation}

The implementation demonstrates that the researchers can create novel MRs and effortlessly use them on DBMS testing using the MTKeras. Our future work will involve applying MTKeras to a specific instance of DBMS. 


\section{Chapter 5}

\section{Conclusion}

In this thesis, to answer RQ1 and RQ2, we have presented MTKeras, a generic and domain-independent, automated MT platform. Besides, we have demonstrated its applicability and problem-detection effectiveness through case studies in five domains (image classification model testing, sentiment analysis model testing, ImageAI testing, search engine testing and database testing). We have also open-sourced the platform and contributed to METWIKI project.

We have shown that the composition of MRs can greatly improve the problem-detection effectiveness of individual MRs, and that MRs can help to enhance a user's understanding of the underlying system(s). We have also found that increasing the number of MRIPs combined in a single MR can increase the violation rate of the MR. The work reported in this thesis has also demonstrated the usefulness of metamorphic relation patterns (MRPs).

On evaluating MTKeras, we find this tool can be useful in terms of communicating the concept of MRP in the research community and broadening the audience of MT. We have held a seminar at dataReachable Pty Ltd ${ }^{\mathrm{a}}$, inviting 5 software testers and 16 software engineers to attend. DataReachable Pty Ltd is a data-driven IT company which focuses on data collection and data processing. The participants had little prior knowledge about MT, but interested in ML testing. Thus, we introduced the MTKeras. After studying the concept of MT and MRP, the participants applied the MTKeras on testing ImageAI application. We next conducted a survey (see Appendix D) among the participants. The majority (14 of 21) of the respondents reflected that MTKeras is simple and highly efficient.

Nevertheless, some of the research reported in this thesis about the usefulness of MTKeras, and MRPs in general, remains at a relatively early stage. Use of MTKeras to test

\footnotetext{
ahttps://www.datareachable.com/
} 
databases needs to be further developed, which we look forward to doing as part of our future work. We wish to promote this tool to the testing community and encourage more contributions from them so that more metamorphic relation patterns and application domains will be supported. We also expect to extend the application of MTKeras to other domains. We look forward to further developing MTKeras and to extend the applicability of MRPs through the MTKeras testing platform. 

[11] T. Y. Chen, F.-C. Kuo, W. Ma, et al., "Metamorphic testing for cybersecurity," Computer, vol. 49, no. 6, pp. 48-55, 2016.

[12] S. Segura, J. A. Parejo, J. Troya, and A. Ruiz-Cortés, "Metamorphic testing of RESTful web APIs," IEEE Transactions on Software Engineering, vol. 44, no. 11, pp. 1083-1099, 2018.

[13] M. Lindvall, A. Porter, G. Magnusson, and C. Schulze, "Metamorphic model-based testing of autonomous systems," in Proceedings of the IEEE/ACM 2nd International Workshop on Metamorphic Testing (MET '17), IEEE, 2017, pp. 35-41.

[14] C. Murphy, G. Kaiser, and M. Arias, "An approach to software testing of machine learning applications," Department of Computer Science, Columbia University, Tech. Rep. CUCS-014-07, 2007.

[15] S. Nakajima, "Generalized oracle for testing machine learning computer programs," in Proceedings of the Software Engineering and Formal Methods (FMSE '18), Springer International Publishing, 2018, pp. 174-179.

[16] J. Ding, X. Kang, and X.-H. Hu, "Validating a deep learning framework by metamorphic testing," in Proceedings of the IEEE/ACM 2nd International Workshop on Metamorphic Testing (MET '17), IEEE, 2017, pp. 28-34.

[17] X. Xie, L. Ma, F. Juefei-Xu, et al., "Deephunter: A coverage-guided fuzz testing framework for deep neural networks," in Proceedings of the 28th ACM SIGSOFT International Symposium on Software Testing and Analysis (ISSTA '19), Beijing, China: ACM, 2019, pp. 146-157.

[18] S. Nagai and T. Tsuchiya, "Applying metamorphic testing to e-commerce product search engines," in Proceedings of the IEEE 23rd Pacific Rim International Symposium on Dependable Computing (PRDC '18), IEEE, 2018, pp. 183-184.

[19] T. E. Oliphant, "Python for scientific computing," Computing in Science Engineering, vol. 9, no. 3, pp. 10-20, 2007.

[20] F. Chollet et al., Keras, 2015. [Online]. Available: https://keras .io.

[21] M. Abadi, P. Barham, J. Chen, et al., "Tensorflow: A system for large-scale machine learning," in Proceedings of the 12th USENIX Symposium on Operating Systems Design and Implementation (OSDI '16), Georgia, USA: USENIX Association, 2016, pp. 265-283.

[22] Moses and J. Olafenwa, ImageAI, an open source Python library built to empower developers to build applications and systems with self-contained computer vision capabilities, 2018. [Online]. Available: https ://github.com/OlafenwaMoses/ ImageAI. 
[23] X. Xie, J. Li, C. Wang, and T. Y. Chen, "Looking for an mr? Try metwiki today," in Proceedings of the IEEE/ACM 1st International Workshop on Metamorphic Testing (MET'16), IEEE, 2016, pp. 1-4.

[24] T. Y. Chen, F.-C. Kuo, H. Liu, et al., "Metamorphic testing: A review of challenges and opportunities," ACM Computing Surveys, vol. 51, no. 1, pp. 1-27, 2018.

[25] T. Y. Chen, P. L. Poon, and X. Xie, "METRIC: Metamorphic relation identification based on the category-choice framework," Journal of Systems and Software, vol. 116, pp. 177-190, 2016.

[26] T. Y. Chen, T. H. Tse, and Y. T. Yu, "Proportional sampling strategy: A compendium and some insights," Journal of Systems and Software, vol. 58, pp. 65$81,2001$.

[27] T. Y. Chen, D. H. Huang, T. H. Tse, and Z. Q. Zhou, "Case studies on the selection of useful relations in metamorphic testing," in Proceedings of the 4th IberoAmerican Symposium on Software Engineering and Knowledge Engineering (JIISIC'04), Madrid, Spain: Polytechnic University of Madrid, 2004, pp. 569-583.

[28] Y. Cao, Z. Q. Zhou, and T. Y. Chen, "On the correlation between the effectiveness of metamorphic relations and dissimilarities of test case executions," in Proceedings of the 13th International Conference on Quality Software (QSIC'13), IEEE, 2013, pp. 153-162.

[29] S. Segura, A. Durán, J. Troya, and A. R. Cortés, "A template-based approach to describing metamorphic relations," in Proceedings of the IEEE/ACM 2nd International Workshop on Metamorphic Testing (MET '17), IEEE, 2017, pp. 3-9.

[30] C. Wu, L. Sun, and Z. Q. Zhou, "The impact of a dot: Case studies of a noise metamorphic relation pattern," in Proceedings of the IEEE/ACM 4th International Workshop on Metamorphic Testing (MET '19), IEEE, 2019, pp. 17-23.

[31] Z. Q. Zhou, T. H. Tse, F.-C. Kuo, and T. Y. Chen, "Automated functional testing of web search engines in the absence of an oracle," Department of Computer Science, The University of Hong Kong, Tech. Rep. TR-2007-06, 2007.

[32] Z. Q. Zhou, S. Zhang, M. Hagenbuchner, T. H. Tse, F.-C. Kuo, and T. Y. Chen, "Automated functional testing of online search services," Software Testing, Verification and Reliability, vol. 22, no. 4, pp. 221-243, 2012.

[33] S. Segura, "Metamorphic testing: Challenges ahead," in Proceedings of the 3rd International Workshop on Metamorphic Testing (MET'18), Gothenburg, Sweden: ACM, 2018, p. 1. 
[34] C. Murphy, G. E. Kaiser, and L. Hu, "Properties of machine learning applications for use in metamorphic testing," Department of Computer Science, Columbia University, Tech. Rep. CUCS-011-08, 2008.

[35] C. Murphy, "Metamorphic testing techniques to detect defects in applications without test oracles," Department of Computer Science, Columbia University, Tech. Rep. CUCS-010-10, 2010.

[36] H. Liu, X. Liu, and T. Y. Chen, "A new method for constructing metamorphic relations," in Proceedings of the 12th International Conference on Quality Software (QSIC '12), IEEE, 2012, pp. 59-68.

[37] X. Xie, J. W. Ho, C. Murphy, G. Kaiser, B. Xu, and T. Y. Chen, "Testing and validating machine learning classifiers by metamorphic testing," Journal of Systems and Software, vol. 84, no. 4, pp. 544-558, 2011.

[38] S. Al-Azani and J. Hassine, "Validation of machine learning classifiers using metamorphic testing and feature selection techniques," in Proceedings of the 11th International Workshop on Multi-disciplinary Trends in Artificial Intelligence (MIWAI '17), Springer International Publishing, 2017, pp. 77-91.

[39] P. Arcaini, A. Bombarda, S. Bonfanti, and A. Gargantini, "Dealing with robustness of convolutional neural networks for image classification," in Proceedings of the 2020 IEEE International Conference on Artificial Intelligence Testing (AITest '20), Oxford, UK: IEEE, 2020, pp. 7-14.

[40] S. Nakajima and H. N. Bui, "Dataset coverage for testing machine learning computer programs," in Proceedings of the 23rd Asia-Pacific Software Engineering Conference (APSEC '16), IEEE, 2016, pp. 297-304.

[41] L. Xu, D. Towey, A. P. French, S. Benford, Z. Q. Zhou, and T. Y. Chen, "Enhancing supervised classifications with metamorphic relations," in Proceedings of the IEEE/ACM 3rd International Workshop on Metamorphic Testing (MET '18), IEEE, 2018, pp. 46-53.

[42] A. Dwarakanath, M. Ahuja, S. Sikand, et al., "Identifying implementation bugs in machine learning based image classifiers using metamorphic testing," in Proceedings of the 27th ACM SIGSOFT International Symposium on Software Testing and Analysis (ISSTA '18), Amsterdam, Netherlands: ACM, 2018, pp. 118-128.

[43] M. Landoni and S. Bell, "Information retrieval techniques for evaluating search engines: a critical overview," Aslib Proceedings, vol. 52, no. 3, pp. 124-129, 2000.

[44] W. Zheng, H. Ma, M. R. Lyu, T. Xie, and I. King, "Mining test oracles of web search engines," in Proceedings of the 26th IEEE/ACM International Conference on Automated Software Engineering (ASE'11), IEEE, 2011, pp. 408-411. 
[45] D. Maier, J. Stein, A. Otis, and A. Purdy, "Development of an object-oriented DBMS," ACM SIGPLAN Notices, vol. 21, no. 11, pp. 472-482, 1986.

[46] L. Giakoumakis and C. Galindo-Legaria, "Testing SQL server's query optimizer: Challenges, techniques and experiences," IEEE Data Engineering Bulletin, vol. 31, pp. 36-43, 2008.

[47] M. Rigger and Z. Su, "Testing database engines via pivoted query synthesis," in Proceedings of the 14th USENIX Symposium on Operating Systems Design and Implementation (OSDI '20), USENIX Association, 2020, pp. 667-682.

[48] D. R. Slutz, "Massive stochastic testing of SQL," in Proceedings of the 24rd International Conference on Very Large Data Bases (VLDB '98), California, USA: Morgan Kaufmann Publishers Inc., 1998, pp. 618-622.

[49] K. Qiu, Z. Zheng, T. Y. Chen, and P.-L. Poon, "Theoretical and empirical analyses of the effectiveness of metamorphic relation composition," IEEE Transactions on Software Engineering, in press, 2020.

[50] L. Sun and Z. Q. Zhou, "Metamorphic testing for machine translations: MT4MT," in Proceedings of the 25th Australasian Software Engineering Conference (ASWEC '18), IEEE, 2018, pp. 96-100.

[51] Y. Lecun, L. Bottou, Y. Bengio, and P. Haffner, "Gradient-based learning applied to document recognition," Proceedings of the IEEE, vol. 86, no. 11, pp. 2278-2324, 1998.

[52] Y. Liu, Y. Liu, T. Y. Chen, and Z. Q. Zhou, "A testing tool for machine learning applications," in Proceedings of the IEEE/ACM 42nd International Conference on Software Engineering Workshops (ICSEW '20), Seoul, Republic of Korea: ACM, 2020, pp. 386-387.

[53] W. Zhang, K. Itoh, J. Tanida, and Y. Ichioka, "Parallel distributed processing model with local space-invariant interconnections and its optical architecture," Applied Optics, vol. 29, no. 32, pp. 4790-4797, 1990.

[54] S. Lai, L. Xu, K. Liu, and J. Zhao, "Recurrent convolutional neural networks for text classification," Proceedings of the Twenty-Ninth AAAI Conference on Artificial Intelligence (AAAI'15), vol. 29, no. 1, 2015.

[55] A. Joulin, E. Grave, P. Bojanowski, and T. Mikolov, "Bag of tricks for efficient text classification," in Proceedings of the 15th Conference of the European Chapter of the Association for Computational Linguistics: Volume 2, Short Papers (ACL '17), Valencia, Spain: ACL, 2017, pp. 427-431. 
[56] F. A. Gers, J. A. Schmidhuber, and F. A. Cummins, "Learning to forget: Continual prediction with LSTM," Neural Computation, vol. 12, no. 10, pp. 2451-2471, 2000 .

[57] X. Huang, P. Wang, X. Cheng, D. Zhou, Q. Geng, and R. Yang, "The apolloscape open dataset for autonomous driving and its application," IEEE Transactions on Pattern Analysis and Machine Intelligence, vol. 42, no. 10, pp. 2702-2719, 2020. 


\section{Appendix A}

\section{A.1 The results of the ImageAI experiment}

The three tables record the experiment results (the number of cars detected in the pictures) of applying ImageAI using three different models (YOLO, RetinaNet, TinyYOLO). "r_5" stands for the MRIP: "rotate 5 degrees"; "b_9" stands for brightness 90\%; "a_10" stands for adding $101 \times 1$ pixel noise. "r_5 b_9" stands for the combination of MRIP "rotate 5 degrees and brightness $90 \% "$ 
Table A.1: The results of experiment using "YOLO" model

\begin{tabular}{|c|c|c|c|c|c|c|c|c|c|}
\hline MRIP & picture1 & picture2 & picture3 & picture4 & picture5 & picture6 & picture7 & picture8 & picture9 \\
\hline$r_{-} 5$ & 7 & 8 & 11 & 8 & 5 & 7 & 7 & 11 & 10 \\
\hline $\mathrm{r}_{-} 10$ & 7 & 8 & 10 & 6 & 7 & 6 & 7 & 13 & 9 \\
\hline$r_{-} 15$ & 6 & 5 & 7 & 7 & 6 & 5 & 6 & 11 & 6 \\
\hline $\mathbf{r}_{-} 20$ & 7 & 5 & 6 & 8 & 6 & 5 & 5 & 9 & 6 \\
\hline b_9 & 7 & 9 & 8 & 8 & 5 & 9 & 7 & 11 & 9 \\
\hline b_8 & 7 & 9 & 8 & 8 & 5 & 9 & 7 & 11 & 9 \\
\hline$b_{-} 7$ & 7 & 9 & 8 & 8 & 5 & 9 & 7 & 11 & 9 \\
\hline b_6 & 7 & 9 & 8 & 8 & 5 & 9 & 7 & 11 & 9 \\
\hline a_10 & 7 & 9 & 8 & 8 & 5 & 9 & 7 & 11 & 9 \\
\hline$a_{-} 100$ & 7 & 9 & 8 & 8 & 5 & 9 & 7 & 11 & 9 \\
\hline a_1000 & 7 & 9 & 8 & 8 & 5 & 9 & 7 & 11 & 9 \\
\hline a_10000 & 7 & 9 & 8 & 8 & 5 & 9 & 7 & 11 & 9 \\
\hline r_5 b_9 & 7 & 8 & 11 & 8 & 5 & 7 & 7 & 11 & 10 \\
\hline $\mathrm{r} \_5 \mathrm{~b} \_8$ & 7 & 8 & 11 & 8 & 5 & 7 & 7 & 11 & 10 \\
\hline r_5 b_7 & 7 & 8 & 11 & 8 & 5 & 7 & 7 & 11 & 10 \\
\hline
\end{tabular}


Table A.1 continued from previous page

\begin{tabular}{|c|c|c|c|c|c|c|c|c|c|}
\hline MRIP & picture1 & picture2 & picture3 & picture4 & picture5 & picture6 & picture7 & picture8 & picture9 \\
\hline r_5 b_6 & 7 & 8 & 11 & 8 & 5 & 7 & 7 & 11 & 10 \\
\hline r_10 b_9 & 7 & 8 & 10 & 6 & 7 & 6 & 7 & 13 & 9 \\
\hline$r_{-} \_10 b \_8$ & 8 & 8 & 10 & 6 & 7 & 6 & 7 & 13 & 9 \\
\hline$r_{-} \_10 b_{-} 7$ & 8 & 8 & 10 & 6 & 7 & 6 & 7 & 13 & 9 \\
\hline r_10 b_6 & 8 & 8 & 10 & 6 & 7 & 6 & 7 & 13 & 9 \\
\hline r_15 b_9 & 7 & 5 & 7 & 7 & 6 & 5 & 6 & 11 & 6 \\
\hline $\mathrm{r} \_15 \mathrm{~b} \_8$ & 7 & 5 & 7 & 7 & 6 & 5 & 6 & 11 & 6 \\
\hline $\mathrm{r}_{-} 15 \mathrm{~b} \_7$ & 7 & 5 & 7 & 7 & 6 & 5 & 6 & 11 & 6 \\
\hline $\mathrm{r}_{-} 15 \mathrm{~b} \_6$ & 7 & 5 & 7 & 7 & 6 & 5 & 6 & 11 & 6 \\
\hline r_20 b_9 & 6 & 5 & 6 & 8 & 6 & 5 & 5 & 9 & 6 \\
\hline $\mathrm{r} \_20 \mathrm{~b} \_8$ & 6 & 5 & 6 & 8 & 6 & 5 & 5 & 9 & 6 \\
\hline $\mathrm{r}_{-} 20 \mathrm{~b} \_7$ & 7 & 5 & 6 & 8 & 6 & 5 & 5 & 9 & 6 \\
\hline r_20 b_6 & 7 & 5 & 6 & 8 & 6 & 5 & 5 & 9 & 6 \\
\hline $\mathrm{r}_{-} 5 \mathrm{a} \_10$ & 7 & 8 & 11 & 8 & 5 & 7 & 7 & 11 & 10 \\
\hline r_5 a_100 & 7 & 8 & 11 & 8 & 5 & 7 & 7 & 11 & 10 \\
\hline
\end{tabular}


Table A.1 continued from previous page

\begin{tabular}{|c|c|c|c|c|c|c|c|c|c|}
\hline MRIP & picture1 & picture2 & picture 3 & picture 4 & picture5 & picture6 & picture7 & picture 8 & picture9 \\
\hline r_5 a_1000 & 7 & 8 & 11 & 8 & 5 & 7 & 7 & 11 & 10 \\
\hline r_5 a_10000 & 7 & 8 & 11 & 8 & 5 & 7 & 7 & 11 & 10 \\
\hline r_10 a_10 & 7 & 8 & 10 & 6 & 7 & 6 & 7 & 13 & 9 \\
\hline$r_{-} 10 a_{-} 100$ & 7 & 8 & 10 & 6 & 7 & 6 & 7 & 13 & 9 \\
\hline$r_{-} 10$ a_1000 & 7 & 8 & 10 & 6 & 7 & 6 & 7 & 13 & 9 \\
\hline r_10 a_10000 & 7 & 8 & 10 & 6 & 7 & 6 & 7 & 13 & 9 \\
\hline $\mathrm{r}_{-} 15 \mathrm{a} \_10$ & 6 & 5 & 7 & 7 & 6 & 5 & 6 & 11 & 6 \\
\hline $\mathrm{r}_{-} 15 \mathrm{a} \_100$ & 6 & 5 & 7 & 7 & 6 & 5 & 6 & 11 & 6 \\
\hline $\mathrm{r}_{-} 15 \mathrm{a} \_1000$ & 6 & 5 & 7 & 7 & 6 & 5 & 6 & 11 & 6 \\
\hline $\mathrm{r}_{-} 15 \mathrm{a}_{-} 10000$ & 6 & 5 & 7 & 7 & 6 & 5 & 6 & 11 & 6 \\
\hline $\mathrm{r} \_20 \mathrm{a} \_10$ & 7 & 5 & 6 & 8 & 6 & 5 & 5 & 9 & 6 \\
\hline$r_{-} 20 a_{-} \_100$ & 7 & 5 & 6 & 8 & 6 & 5 & 5 & 9 & 6 \\
\hline r_ 20 a_1 1000 & 7 & 5 & 6 & 8 & 6 & 5 & 5 & 9 & 6 \\
\hline r_20 a_10000 & 7 & 5 & 6 & 8 & 6 & 5 & 5 & 9 & 6 \\
\hline b_9 a_10 & 7 & 9 & 8 & 8 & 5 & 9 & 7 & 11 & 9 \\
\hline
\end{tabular}


Table A.1 continued from previous page

\begin{tabular}{|c|c|c|c|c|c|c|c|c|c|}
\hline MRIP & picture 1 & picture2 & picture3 & picture 4 & picture5 & picture6 & picture7 & picture 8 & picture9 \\
\hline b_9 a_100 & 7 & 9 & 8 & 8 & 5 & 9 & 7 & 11 & 9 \\
\hline b_9 a_1000 & 7 & 9 & 8 & 8 & 5 & 9 & 7 & 11 & 9 \\
\hline | b_9 a_10000 & 7 & 9 & 8 & 8 & 5 & 9 & 7 & 11 & 9 \\
\hline b_8 a_10 & 7 & 9 & 8 & 8 & 5 & 9 & 7 & 11 & 9 \\
\hline b_8 a_100 & 7 & 9 & 8 & 8 & 5 & 9 & 7 & 11 & 9 \\
\hline b_8 a_1000 & 7 & 9 & 8 & 8 & 5 & 9 & 7 & 11 & 9 \\
\hline b_8 a_10000 & 7 & 9 & 8 & 8 & 5 & 9 & 7 & 11 & 9 \\
\hline$b_{-}$7 $a_{-} \_10$ & 7 & 9 & 8 & 8 & 5 & 9 & 7 & 11 & 9 \\
\hline b_7 a_100 & 7 & 9 & 8 & 8 & 5 & 9 & 7 & 11 & 9 \\
\hline b_7 a_1000 & 7 & 9 & 8 & 8 & 5 & 9 & 7 & 11 & 9 \\
\hline $\mathrm{b}_{-}$7 $\mathrm{a}_{-} 10000$ & 7 & 9 & 8 & 8 & 5 & 9 & 7 & 11 & 9 \\
\hline b_6 a_10 & 7 & 9 & 8 & 8 & 5 & 9 & 7 & 11 & 9 \\
\hline b_6 a_100 & 7 & 9 & 8 & 8 & 5 & 9 & 7 & 11 & 9 \\
\hline b_6 a_1000 & 7 & 9 & 8 & 8 & 5 & 9 & 7 & 11 & 9 \\
\hline b_6 a_10000 & 7 & 9 & 8 & 8 & 5 & 9 & 7 & 11 & 9 \\
\hline
\end{tabular}


Table A.1 continued from previous page

\begin{tabular}{|c|c|c|c|c|c|c|c|c|c|}
\hline MRIP & picture1 & picture2 & picture3 & picture4 & picture5 & picture6 & picture7 & picture 8 & picture9 \\
\hline b_9 r_5 a_10 & 7 & 8 & 11 & 8 & 5 & 7 & 7 & 11 & 10 \\
\hline b_9 r_5 a_100 & 7 & 8 & 11 & 8 & 5 & 7 & 7 & 11 & 10 \\
\hline b_9 r_5 a_1000 & 7 & 8 & 11 & 8 & 5 & 7 & 7 & 11 & 10 \\
\hline b_9 r_5 a_10000 & 7 & 8 & 11 & 8 & 5 & 7 & 7 & 11 & 10 \\
\hline b_9 r_10 a_10 & 7 & 8 & 10 & 6 & 7 & 6 & 7 & 13 & 9 \\
\hline b_9 r_10 a_100 & 7 & 8 & 10 & 6 & 7 & 6 & 7 & 13 & 9 \\
\hline b_9 r_10 a_1000 & 7 & 8 & 10 & 6 & 7 & 6 & 7 & 13 & 9 \\
\hline b_9 r_10 a_10000 & 7 & 8 & 10 & 6 & 7 & 6 & 7 & 13 & 9 \\
\hline b_9 r_15 a_10 & 7 & 5 & 7 & 7 & 6 & 5 & 6 & 11 & 6 \\
\hline $\mathrm{b}_{-} 9 \mathrm{r}_{-} 15$ a_-100 & 7 & 5 & 7 & 7 & 6 & 5 & 6 & 11 & 6 \\
\hline b_9 r_15 a_1000 & 7 & 5 & 7 & 7 & 6 & 5 & 6 & 11 & 6 \\
\hline b_9 r_15 a_10000 & 7 & 5 & 7 & 7 & 6 & 5 & 6 & 11 & 6 \\
\hline b_9 r_20 a_10 & 6 & 5 & 6 & 8 & 6 & 5 & 5 & 9 & 6 \\
\hline b_9 r_20 a $\_100$ & 6 & 5 & 6 & 8 & 6 & 5 & 5 & 9 & 6 \\
\hline b_9 r_20 a_1000 & 6 & 5 & 6 & 8 & 6 & 5 & 5 & 9 & 6 \\
\hline
\end{tabular}


Table A.1 continued from previous page

\begin{tabular}{|c|c|c|c|c|c|c|c|c|c|}
\hline MRIP & picture 1 & picture2 & picture3 & picture4 & picture5 & picture6 & picture7 & picture 8 & picture9 \\
\hline b_9 r_20 a_10000 & 6 & 5 & 6 & 8 & 6 & 5 & 5 & 9 & 6 \\
\hline b_8 r_5 a_10 & 7 & 8 & 11 & 8 & 5 & 7 & 7 & 11 & 10 \\
\hline b_8 r_5 a_100 & 7 & 8 & 11 & 8 & 5 & 7 & 7 & 11 & 10 \\
\hline b_8 r_5 a_1000 & 7 & 8 & 11 & 8 & 5 & 7 & 7 & 11 & 10 \\
\hline b_8 r_5 a_10000 & 7 & 8 & 11 & 8 & 5 & 7 & 7 & 11 & 10 \\
\hline b_8 r_10 a_10 & 8 & 8 & 10 & 6 & 7 & 6 & 7 & 13 & 9 \\
\hline b_8 $\mathrm{r}_{-} 10 \mathrm{a} \_100$ & 8 & 8 & 10 & 6 & 7 & 6 & 7 & 13 & 9 \\
\hline b_8 r_10 a_1000 & 8 & 8 & 10 & 6 & 7 & 6 & 7 & 13 & 9 \\
\hline b_8 r_10 a_10000 & 8 & 8 & 10 & 6 & 7 & 6 & 7 & 13 & 9 \\
\hline b_8 $r_{-} 15$ a_10 & 7 & 5 & 7 & 7 & 6 & 5 & 6 & 11 & 6 \\
\hline b_8 r $\_15$ a $\_100$ & 7 & 5 & 7 & 7 & 6 & 5 & 6 & 11 & 6 \\
\hline b_8 r_15 a_1000 & 7 & 5 & 7 & 7 & 6 & 5 & 6 & 11 & 6 \\
\hline b_8 r_15 a_10000 & 7 & 5 & 7 & 7 & 6 & 5 & 6 & 11 & 6 \\
\hline b_8 $r \_20$ a $\_10$ & 6 & 5 & 6 & 8 & 6 & 5 & 5 & 9 & 6 \\
\hline b_8 $r \_20$ a $\_100$ & 6 & 5 & 6 & 8 & 6 & 5 & 5 & 9 & 6 \\
\hline
\end{tabular}


Table A.1 continued from previous page

\begin{tabular}{|c|c|c|c|c|c|c|c|c|c|}
\hline MRIP & picture1 & picture2 & picture3 & picture4 & picture5 & picture6 & picture7 & picture 8 & picture9 \\
\hline b_8 $r \_20$ a_1000 & 6 & 5 & 6 & 8 & 6 & 5 & 5 & 9 & 6 \\
\hline b_8 r_20 a_10000 & 6 & 5 & 6 & 8 & 6 & 5 & 5 & 9 & 6 \\
\hline b_7 r_5 a_10 & 7 & 8 & 11 & 8 & 5 & 7 & 7 & 11 & 10 \\
\hline b_7 r_5 a_100 & 7 & 8 & 11 & 8 & 5 & 7 & 7 & 11 & 10 \\
\hline b_7 r_5 a_1000 & 7 & 8 & 11 & 8 & 5 & 7 & 7 & 11 & 10 \\
\hline b_7 r_5 a_10000 & 7 & 8 & 11 & 8 & 5 & 7 & 7 & 11 & 10 \\
\hline $\mathrm{b}_{-} 7 \mathrm{r} \_10 \mathrm{a} \_10$ & 8 & 8 & 10 & 6 & 7 & 6 & 7 & 13 & 9 \\
\hline $\mathrm{b}_{-} 7 \mathrm{r} \_10 \mathrm{a}_{-} 100$ & 8 & 8 & 10 & 6 & 7 & 6 & 7 & 13 & 9 \\
\hline $\mathrm{b}_{-}$7 $\mathrm{r}_{-} 10 \mathrm{a} \_1000$ & 8 & 8 & 10 & 6 & 7 & 6 & 7 & 13 & 9 \\
\hline $\mathrm{b}_{-} 7 \mathrm{r}_{-} 10 \mathrm{a}_{-} 10000$ & 8 & 8 & 10 & 6 & 7 & 6 & 7 & 13 & 9 \\
\hline b_7 r_15 a_10 & 7 & 5 & 7 & 7 & 6 & 5 & 6 & 11 & 6 \\
\hline $\mathrm{b}_{-} 7 \mathrm{r} \_15 \mathrm{a} \_100$ & 7 & 5 & 7 & 7 & 6 & 5 & 6 & 11 & 6 \\
\hline $\mathrm{b}_{-} 7 \mathrm{r} \_15 \mathrm{a} \_1000$ & 7 & 5 & 7 & 7 & 6 & 5 & 6 & 11 & 6 \\
\hline $\mathrm{b}_{-} 7 \mathrm{r}_{-} 15$ a_10000 & 7 & 5 & 7 & 7 & 6 & 5 & 6 & 11 & 6 \\
\hline $\mathrm{b}_{-} 7 \mathrm{r} \_20 \mathrm{a} \_10$ & 7 & 5 & 6 & 8 & 6 & 5 & 5 & 9 & 6 \\
\hline
\end{tabular}


Table A.1 continued from previous page

\begin{tabular}{|c|c|c|c|c|c|c|c|c|c|}
\hline MRIP & picture1 & picture2 & picture3 & picture4 & picture5 & picture6 & picture7 & picture8 & picture9 \\
\hline b_7 r_20 a_100 & 7 & 5 & 6 & 8 & 6 & 5 & 5 & 9 & 6 \\
\hline b_7 r_20 a_1000 & 7 & 5 & 6 & 8 & 6 & 5 & 5 & 9 & 6 \\
\hline b_7 r_20 a_10000 & 7 & 5 & 6 & 8 & 6 & 5 & 5 & 9 & 6 \\
\hline b_6 r_5 a_10 & 7 & 8 & 11 & 8 & 5 & 7 & 7 & 11 & 10 \\
\hline b_6 r_5 a_100 & 7 & 8 & 11 & 8 & 5 & 7 & 7 & 11 & 10 \\
\hline b_6 r_5 a_1000 & 7 & 8 & 11 & 8 & 5 & 7 & 7 & 11 & 10 \\
\hline b_6 r $\_5$ a $\_10000$ & 7 & 8 & 11 & 8 & 5 & 7 & 7 & 11 & 10 \\
\hline b_6 r $\_10$ a_10 & 8 & 8 & 10 & 6 & 7 & 6 & 7 & 13 & 9 \\
\hline b_6 r $\_10$ a $\_100$ & 8 & 8 & 10 & 6 & 7 & 6 & 7 & 13 & 9 \\
\hline b_6 r_10 a_1000 & 8 & 8 & 10 & 6 & 7 & 6 & 7 & 13 & 9 \\
\hline b_6 r_10 a_10000 & 8 & 8 & 10 & 6 & 7 & 6 & 7 & 13 & 9 \\
\hline b_6 r $\_15$ a__10 & 7 & 5 & 7 & 7 & 6 & 5 & 6 & 11 & 6 \\
\hline b_6 r $\_15$ a $\_100$ & 7 & 5 & 7 & 7 & 6 & 5 & 6 & 11 & 6 \\
\hline b_6 r $\_15$ a_1000 & 7 & 5 & 7 & 7 & 6 & 5 & 6 & 11 & 6 \\
\hline b_6 r_15 a_10000 & 7 & 5 & 7 & 7 & 6 & 5 & 6 & 11 & 6 \\
\hline
\end{tabular}


Table A.1 continued from previous page

\begin{tabular}{|c|c|c|c|c|c|c|c|c|c|}
\hline MRIP & picture 1 & picture2 & picture3 & picture 4 & picture5 & picture6 & picture7 & picture8 & picture9 \\
\hline b_6 r_20 a_10 & 7 & 5 & 6 & 8 & 6 & 5 & 5 & 9 & 6 \\
\hline b_6 r_20 a_100 & 7 & 5 & 6 & 8 & 6 & 5 & 5 & 9 & 6 \\
\hline b_6 r_20 a_1000 & 7 & 5 & 6 & 8 & 6 & 5 & 5 & 9 & 6 \\
\hline b_6 r_20 a_10000 & 7 & 5 & 6 & 8 & 6 & 5 & 5 & 9 & 6 \\
\hline
\end{tabular}


Table A.2: The results of experiment using "RetinaNet" model

\begin{tabular}{|c|c|c|c|c|c|c|c|c|c|}
\hline MRIP & picture 1 & picture2 & picture 3 & picture4 & picture5 & picture6 & picture7 & picture8 & picture9 \\
\hline r_5 & 9 & 4 & 10 & 8 & 5 & 7 & 22 & 15 & 5 \\
\hline $\mathrm{r}_{-} 10$ & 11 & 5 & 12 & 6 & 8 & 6 & 20 & 16 & 6 \\
\hline $\mathrm{r}_{-} 15$ & 8 & 4 & 8 & 8 & 6 & 6 & 19 & 18 & 7 \\
\hline r_20 & 5 & 4 & 6 & 11 & 8 & 8 & 13 & 17 & 5 \\
\hline b_9 & 7 & 6 & 17 & 9 & 10 & 6 & 20 & 15 & 6 \\
\hline b_8 & 7 & 6 & 17 & 9 & 10 & 6 & 20 & 15 & 6 \\
\hline$b_{-} 7$ & 7 & 6 & 17 & 9 & 10 & 6 & 20 & 15 & 6 \\
\hline b_6 & 7 & 6 & 17 & 9 & 10 & 6 & 20 & 15 & 6 \\
\hline$a_{-} 10$ & 7 & 6 & 17 & 9 & 10 & 6 & 20 & 15 & 6 \\
\hline a_100 & 7 & 6 & 17 & 9 & 10 & 6 & 20 & 15 & 6 \\
\hline$a_{-} 1000$ & 7 & 6 & 17 & 9 & 10 & 6 & 21 & 15 & 6 \\
\hline$a_{-} 10000$ & 6 & 6 & 18 & 11 & 9 & 6 & 20 & 14 & 6 \\
\hline violations & 5 & 4 & 5 & 5 & 5 & 2 & 4 & 4 & 3 \\
\hline r_5 b_9 & 10 & 4 & 10 & 8 & 5 & 7 & 22 & 15 & 5 \\
\hline r_5 b_8 & 10 & 4 & 10 & 8 & 5 & 7 & 22 & 15 & 5 \\
\hline
\end{tabular}


Table A.2 continued from previous page

\begin{tabular}{|c|c|c|c|c|c|c|c|c|c|}
\hline MRIP & picture 1 & picture 2 & picture 3 & picture4 & picture 5 & picture6 & picture 7 & picture 8 & picture9 \\
\hline r_5 b_7 & 10 & 4 & 10 & 8 & 5 & 7 & 22 & 15 & 5 \\
\hline r_5 b_6 & 10 & 4 & 10 & 8 & 5 & 7 & 22 & 15 & 5 \\
\hline r_10 b_9 & 11 & 5 & 12 & 6 & 8 & 6 & 20 & 16 & 6 \\
\hline r_10 b_8 & 11 & 5 & 12 & 6 & 8 & 6 & 20 & 16 & 6 \\
\hline$r_{-} 10 \quad b_{-}$7 & 11 & 5 & 12 & 6 & 8 & 6 & 20 & 16 & 6 \\
\hline r_10 b_6 & 12 & 5 & 12 & 6 & 8 & 6 & 20 & 16 & 6 \\
\hline $\mathrm{r}_{-} 15 \mathrm{~b} \_9$ & 7 & 4 & 8 & 8 & 6 & 6 & 19 & 18 & 7 \\
\hline $\mathrm{r}_{-} 15 \mathrm{~b} \_8$ & 7 & 4 & 8 & 8 & 6 & 6 & 19 & 18 & 7 \\
\hline $\mathrm{r}_{-} 15 \mathrm{~b} \_7$ & 7 & 4 & 8 & 8 & 6 & 6 & 19 & 18 & 7 \\
\hline $\mathrm{r}_{-} 15 \mathrm{~b} \_6$ & 7 & 4 & 8 & 8 & 6 & 6 & 19 & 18 & 7 \\
\hline $\mathrm{r}_{-} 20 \mathrm{~b} \_9$ & 5 & 4 & 6 & 11 & 8 & 8 & 13 & 17 & 5 \\
\hline $\mathrm{r} \_20 \mathrm{~b} \_8$ & 5 & 4 & 6 & 11 & 8 & 8 & 13 & 17 & 5 \\
\hline $\mathrm{r}_{-} 20 \mathrm{~b} \_7$ & 5 & 4 & 6 & 11 & 8 & 8 & 13 & 17 & 5 \\
\hline r_20 b_6 & 5 & 4 & 6 & 11 & 8 & 8 & 13 & 17 & 5 \\
\hline r_5 a_10 & 9 & 4 & 10 & 8 & 5 & 7 & 22 & 15 & 5 \\
\hline
\end{tabular}


Table A.2 continued from previous page

\begin{tabular}{|c|c|c|c|c|c|c|c|c|c|}
\hline MRIP & picture 1 & picture 2 & picture 3 & picture4 & picture 5 & picture6 & picture 7 & picture 8 & picture9 \\
\hline r_5 a_100 & 9 & 4 & 10 & 8 & 5 & 7 & 22 & 15 & 5 \\
\hline r_5 a_1000 & 9 & 4 & 10 & 8 & 5 & 7 & 22 & 15 & 5 \\
\hline r_5 a_10000 & 8 & 4 & 11 & 7 & 5 & 7 & 23 & 15 & 5 \\
\hline $\mathrm{r}_{-} 10 \mathrm{a} \_10$ & 11 & 5 & 12 & 6 & 8 & 6 & 20 & 16 & 6 \\
\hline r_10 a_100 & 11 & 5 & 12 & 6 & 8 & 6 & 20 & 16 & 6 \\
\hline r_10 a_1000 & 11 & 5 & 12 & 6 & 8 & 6 & 19 & 17 & 6 \\
\hline $\mathrm{r}_{-} 10 \mathrm{a} \_10000$ & 12 & 6 & 11 & 6 & 8 & 6 & 21 & 15 & 6 \\
\hline $\mathrm{r}_{-} 15 \mathrm{a} \_10$ & 8 & 4 & 8 & 8 & 6 & 6 & 19 & 18 & 7 \\
\hline $\mathrm{r}_{-} 15 \mathrm{a}_{-} 100$ & 8 & 4 & 8 & 8 & 6 & 6 & 19 & 18 & 7 \\
\hline$r_{-} 15$ a_1000 & 8 & 4 & 8 & 8 & 6 & 6 & 19 & 18 & 6 \\
\hline$r_{-} 15$ a_1 10000 & 8 & 4 & 7 & 8 & 6 & 6 & 20 & 18 & 6 \\
\hline $\mathrm{r}_{-} 20 \mathrm{a} \_10$ & 5 & 4 & 6 & 11 & 8 & 8 & 13 & 17 & 5 \\
\hline$r_{-} 20 a_{-} 100$ & 5 & 4 & 6 & 10 & 8 & 8 & 13 & 17 & 5 \\
\hline r_20 a_1000 & 5 & 4 & 6 & 11 & 8 & 8 & 13 & 17 & 5 \\
\hline r_20 a_10000 & 6 & 4 & 7 & 11 & 7 & 8 & 13 & 17 & 4 \\
\hline
\end{tabular}


Table A.2 continued from previous page

\begin{tabular}{|c|c|c|c|c|c|c|c|c|c|}
\hline MRIP & picture 1 & picture2 & picture 3 & picture4 & picture 5 & picture6 & picture 7 & picture 8 & picture9 \\
\hline b_9 a_10 & 7 & 6 & 17 & 9 & 10 & 6 & 20 & 15 & 6 \\
\hline b_9 a_100 & 7 & 6 & 17 & 9 & 10 & 6 & 20 & 15 & 6 \\
\hline b_9 a_1000 & 7 & 6 & 17 & 9 & 10 & 6 & 20 & 14 & 6 \\
\hline b_9 a_10000 & 6 & 6 & 16 & 11 & 10 & 6 & 20 & 15 & 6 \\
\hline b_8 a_10 & 7 & 6 & 17 & 9 & 10 & 6 & 20 & 15 & 6 \\
\hline b_8 a_100 & 7 & 6 & 17 & 9 & 10 & 6 & 21 & 15 & 6 \\
\hline b_8 a_1000 & 7 & 6 & 17 & 9 & 10 & 6 & 21 & 15 & 6 \\
\hline b_8 a_10000 & 6 & 6 & 16 & 8 & 9 & 5 & 20 & 15 & 6 \\
\hline$b_{-} 7 \mathrm{a} \_10$ & 7 & 6 & 17 & 9 & 10 & 6 & 20 & 15 & 6 \\
\hline b_7 a_100 & 7 & 6 & 17 & 9 & 10 & 7 & 20 & 15 & 6 \\
\hline b_7 a_1000 & 7 & 6 & 17 & 9 & 10 & 6 & 21 & 15 & 6 \\
\hline $\mathrm{b}_{-} 7 \mathrm{a}_{-} 10000$ & 7 & 6 & 17 & 11 & 9 & 6 & 21 & 13 & 6 \\
\hline b_6 a_10 & 7 & 6 & 17 & 9 & 10 & 6 & 20 & 15 & 6 \\
\hline b_6 a_100 & 7 & 6 & 17 & 9 & 10 & 6 & 21 & 15 & 6 \\
\hline b_6 a_1000 & 7 & 6 & 17 & 9 & 10 & 6 & 21 & 15 & 6 \\
\hline
\end{tabular}


Table A.2 continued from previous page

\begin{tabular}{|c|c|c|c|c|c|c|c|c|c|}
\hline MRIP & picture 1 & picture 2 & picture 3 & picture4 & picture 5 & picture6 & picture 7 & picture 8 & picture9 \\
\hline b_6 a_10000 & 7 & 6 & 16 & 9 & 10 & 7 & 21 & 14 & 6 \\
\hline violations & 30 & 31 & 35 & 35 & 34 & 19 & 32 & 26 & 22 \\
\hline b_9 r_5 a_10 & 10 & 4 & 10 & 8 & 5 & 7 & 22 & 15 & 5 \\
\hline b_9 r_5 a_100 & 10 & 4 & 10 & 8 & 5 & 7 & 22 & 15 & 5 \\
\hline b_9 r_5 a_1000 & 10 & 4 & 10 & 8 & 5 & 7 & 22 & 15 & 6 \\
\hline b_9 r_5 a_10000 & 9 & 4 & 11 & 9 & 5 & 7 & 21 & 16 & 5 \\
\hline b_9 r_10 a_10 & 11 & 5 & 12 & 6 & 8 & 6 & 20 & 16 & 6 \\
\hline b_9 r_10 a_100 & 11 & 5 & 12 & 6 & 8 & 6 & 20 & 16 & 6 \\
\hline b_9 r_10 a_1000 & 11 & 5 & 12 & 6 & 8 & 6 & 20 & 16 & 6 \\
\hline b_9 r_10 a_10000 & 11 & 6 & 12 & 5 & 8 & 6 & 21 & 16 & 6 \\
\hline b_9 r_15 a_10 & 7 & 4 & 8 & 8 & 6 & 6 & 19 & 18 & 7 \\
\hline b_9 r_15 a_-100 & 7 & 4 & 8 & 8 & 6 & 6 & 19 & 18 & 7 \\
\hline b_9 r_15 a $\_1000$ & 7 & 4 & 8 & 8 & 6 & 6 & 19 & 19 & 6 \\
\hline b_9 r_15 a_10000 & 5 & 3 & 6 & 5 & 6 & 7 & 15 & 20 & 3 \\
\hline b_9 r_20 a_10 & 5 & 4 & 6 & 11 & 8 & 8 & 13 & 17 & 5 \\
\hline
\end{tabular}


Table A.2 continued from previous page

\begin{tabular}{|c|c|c|c|c|c|c|c|c|c|}
\hline MRIP & picture 1 & picture 2 & picture 3 & picture4 & picture 5 & picture6 & picture 7 & picture 8 & picture9 \\
\hline b_9 r_20 a_100 & 6 & 5 & 6 & 8 & 6 & 5 & 5 & 9 & 6 \\
\hline b_9 r_20 a_1000 & 5 & 4 & 6 & 11 & 8 & 8 & 13 & 17 & 5 \\
\hline b_9 r_20 a_10000 & 5 & 4 & 6 & 11 & 8 & 8 & 13 & 17 & 5 \\
\hline b_8 r_5 a_10 & 10 & 4 & 10 & 8 & 5 & 7 & 22 & 15 & 5 \\
\hline b_8 r_5 a_100 & 10 & 4 & 10 & 8 & 5 & 7 & 22 & 15 & 5 \\
\hline b_8 r_5 a_1000 & 10 & 4 & 10 & 7 & 5 & 7 & 22 & 15 & 5 \\
\hline b_8 r_5 a _10000 & 11 & 4 & 10 & 8 & 5 & 7 & 20 & 18 & 6 \\
\hline b_8 $\mathrm{r}_{-} 10 \mathrm{a} \_10$ & 11 & 5 & 12 & 6 & 8 & 6 & 20 & 16 & 6 \\
\hline b_8 $\mathrm{r}_{-} 10 \mathrm{a} \_100$ & 11 & 5 & 12 & 6 & 8 & 6 & 20 & 16 & 6 \\
\hline b_8 r_10 a $\_1000$ & 11 & 5 & 12 & 6 & 8 & 6 & 20 & 17 & 6 \\
\hline b_8 r_10 a_10000 & 11 & 5 & 11 & 7 & 8 & 6 & 21 & 17 & 6 \\
\hline b_8 r_15 a_10 & 7 & 4 & 8 & 8 & 6 & 6 & 19 & 18 & 7 \\
\hline $\mathrm{b} \_8 \mathrm{r} \_15$ a $\_100$ & 7 & 4 & 8 & 8 & 6 & 6 & 19 & 18 & 7 \\
\hline b_8 r_15 a_1000 & 7 & 4 & 8 & 8 & 6 & 6 & 19 & 19 & 6 \\
\hline b_8 r_15 a_10000 & 7 & 4 & 8 & 9 & 6 & 6 & 19 & 20 & 7 \\
\hline
\end{tabular}


Table A.2 continued from previous page

\begin{tabular}{|c|c|c|c|c|c|c|c|c|c|}
\hline MRIP & picture 1 & picture 2 & picture 3 & picture4 & picture 5 & picture6 & picture 7 & picture 8 & picture9 \\
\hline b_8 r_20 a_10 & 5 & 4 & 6 & 11 & 8 & 8 & 13 & 17 & 5 \\
\hline b_8 r_20 a_100 & 5 & 4 & 6 & 10 & 8 & 8 & 13 & 17 & 5 \\
\hline b_8 r_20 a_1000 & 5 & 4 & 6 & 11 & 8 & 8 & 13 & 17 & 5 \\
\hline b_8 r_20 a_10000 & 5 & 4 & 6 & 10 & 8 & 8 & 13 & 18 & 4 \\
\hline b_7 r_5 a_10 & 10 & 4 & 10 & 8 & 5 & 7 & 22 & 15 & 5 \\
\hline b_7 r_5 a_100 & 10 & 4 & 10 & 8 & 5 & 7 & 22 & 15 & 5 \\
\hline b_7 r_5 a_1000 & 10 & 4 & 11 & 8 & 5 & 7 & 22 & 16 & 5 \\
\hline b_7 r_5 a $\_10000$ & 10 & 4 & 10 & 8 & 5 & 7 & 21 & 15 & 6 \\
\hline $\mathrm{b}_{-} 7 \mathrm{r} \_10 \mathrm{a} \_10$ & 11 & 5 & 12 & 6 & 8 & 6 & 20 & 16 & 6 \\
\hline $\mathrm{b} \__{-} 7 \mathrm{r}_{-} 10 \mathrm{a}_{-} 100$ & 11 & 5 & 12 & 6 & 8 & 6 & 20 & 16 & 6 \\
\hline $\mathrm{b}_{-} 7 \mathrm{r} \_10 \mathrm{a} \_1000$ & 11 & 5 & 12 & 6 & 8 & 6 & 20 & 17 & 6 \\
\hline b_7 r_10 a_10000 & 11 & 6 & 12 & 7 & 8 & 6 & 20 & 16 & 6 \\
\hline b_7 r_15 a_10 & 7 & 4 & 8 & 8 & 6 & 6 & 19 & 18 & 7 \\
\hline $\mathrm{b} \__{-} 7 \mathrm{r} \_15 \mathrm{a} \_100$ & 7 & 4 & 8 & 8 & 6 & 6 & 19 & 18 & 6 \\
\hline b_7 r_15 a_1000 & 7 & 4 & 8 & 8 & 6 & 6 & 19 & 18 & 6 \\
\hline
\end{tabular}


Table A.2 continued from previous page

\begin{tabular}{|c|c|c|c|c|c|c|c|c|c|}
\hline MRIP & picture 1 & picture 2 & picture 3 & picture4 & picture 5 & picture6 & picture 7 & picture 8 & picture9 \\
\hline b_7 r_15 a_10000 & 7 & 4 & 8 & 7 & 6 & 6 & 21 & 17 & 6 \\
\hline b_7 r_20 a_10 & 5 & 4 & 6 & 11 & 8 & 8 & 13 & 17 & 5 \\
\hline b_7 r_20 a_100 & 5 & 4 & 6 & 11 & 8 & 8 & 13 & 17 & 5 \\
\hline b_7 r_20 a_1000 & 5 & 4 & 6 & 11 & 8 & 8 & 13 & 17 & 5 \\
\hline b_7 r_20 a_10000 & 5 & 4 & 6 & 11 & 8 & 8 & 13 & 17 & 5 \\
\hline b_6 r_5 a_10 & 10 & 4 & 10 & 8 & 5 & 7 & 22 & 15 & 5 \\
\hline b_6 r 55 a_100 & 10 & 4 & 10 & 8 & 5 & 7 & 22 & 15 & 5 \\
\hline b_6 r_5 a_1000 & 10 & 4 & 10 & 8 & 5 & 7 & 22 & 15 & 5 \\
\hline b_6 r $\_5$ a $\_10000$ & 10 & 4 & 10 & 7 & 5 & 8 & 21 & 14 & 6 \\
\hline b_6 r_10 a $\_10$ & 12 & 5 & 12 & 6 & 8 & 6 & 20 & 16 & 6 \\
\hline b_6 r_10 a_100 & 12 & 5 & 12 & 6 & 8 & 6 & 20 & 16 & 6 \\
\hline b_6 r_10 a $\_1000$ & 12 & 5 & 12 & 6 & 8 & 6 & 19 & 16 & 6 \\
\hline b_6 r_10 a_10000 & 12 & 5 & 13 & 7 & 8 & 6 & 20 & 17 & 6 \\
\hline b_6 r_15 a_10 & 7 & 4 & 8 & 8 & 6 & 6 & 19 & 18 & 7 \\
\hline b_6 r_15 a_100 & 7 & 4 & 8 & 8 & 6 & 6 & 19 & 18 & 7 \\
\hline
\end{tabular}


Table A.2 continued from previous page

\begin{tabular}{|c|c|c|c|c|c|c|c|c|c|}
\hline MRIP & picture1 & picture2 & picture 3 & picture4 & picture 5 & picture6 & picture7 & picture 8 & picture9 \\
\hline b_6 r_15 a_1000 & 7 & 4 & 7 & 8 & 6 & 6 & 19 & 18 & 7 \\
\hline b_6 r_15 a_10000 & 7 & 4 & 7 & 9 & 6 & 6 & 20 & 18 & 6 \\
\hline b_6 r_20 a_10 & 5 & 4 & 6 & 11 & 8 & 8 & 13 & 17 & 5 \\
\hline b_6 r_20 a_100 & 5 & 4 & 6 & 11 & 8 & 8 & 13 & 17 & 5 \\
\hline b_6 r_20 a_1000 & 5 & 4 & 6 & 11 & 8 & 8 & 13 & 17 & 5 \\
\hline b_6 r_20 a_10000 & 5 & 4 & 6 & 11 & 8 & 8 & 13 & 16 & 4 \\
\hline
\end{tabular}


Table A.3: The results of experiment using "Tiny YOLO" model

\begin{tabular}{|c|c|c|c|c|c|c|c|c|c|}
\hline MRIP & picture 1 & picture 2 & picture 3 & picture4 & picture 5 & picture6 & picture7 & picture8 & picture9 \\
\hline r_5 & 8 & 5 & 6 & 6 & 4 & 4 & 9 & 5 & 2 \\
\hline $\mathrm{r}_{-} 10$ & 5 & 3 & 4 & 4 & 4 & 6 & 8 & 10 & 4 \\
\hline$r_{-} 15$ & 7 & 3 & 3 & 4 & 3 & 5 & 10 & 6 & 1 \\
\hline r_20 & 5 & 3 & 2 & 3 & 5 & 4 & 3 & 7 & 2 \\
\hline b_9 & 6 & 3 & 4 & 6 & 4 & 7 & 11 & 6 & 2 \\
\hline b_8 & 6 & 3 & 4 & 6 & 4 & 7 & 11 & 6 & 2 \\
\hline$b_{-} 7$ & 6 & 3 & 4 & 6 & 4 & 7 & 11 & 6 & 2 \\
\hline b_6 & 6 & 3 & 4 & 6 & 4 & 7 & 11 & 6 & 2 \\
\hline$a_{-} 10$ & 7 & 3 & 4 & 6 & 4 & 7 & 11 & 6 & 2 \\
\hline a_100 & 7 & 3 & 4 & 6 & 4 & 7 & 11 & 6 & 2 \\
\hline$a_{-} 1000$ & 7 & 3 & 4 & 6 & 4 & 7 & 11 & 6 & 2 \\
\hline$a_{-} 10000$ & 7 & 3 & 4 & 6 & 4 & 7 & 11 & 6 & 2 \\
\hline r_5 b_9 & 7 & 5 & 6 & 6 & 4 & 4 & 9 & 5 & 2 \\
\hline $\mathrm{r}_{-} 5 \mathrm{~b} \_8$ & 8 & 5 & 6 & 6 & 4 & 4 & 9 & 5 & 2 \\
\hline r_5 b_7 & 6 & 5 & 6 & 6 & 4 & 4 & 9 & 5 & 2 \\
\hline
\end{tabular}


Table A.3 continued from previous page

\begin{tabular}{|c|c|c|c|c|c|c|c|c|c|}
\hline MRIP & picture1 & picture2 & picture 3 & picture4 & picture 5 & picture6 & picture7 & picture8 & picture9 \\
\hline r_5 b_6 & 6 & 5 & 6 & 6 & 4 & 4 & 9 & 5 & 2 \\
\hline r_10 b_9 & 5 & 3 & 4 & 4 & 4 & 6 & 8 & 10 & 4 \\
\hline r_10 b_8 & 5 & 3 & 4 & 4 & 4 & 6 & 8 & 10 & 4 \\
\hline$r_{-} 10 \quad b_{-} 7$ & 5 & 3 & 4 & 4 & 4 & 6 & 8 & 10 & 4 \\
\hline r_10 b_6 & 6 & 3 & 4 & 4 & 4 & 6 & 8 & 10 & 4 \\
\hline r_15 b_9 & 6 & 3 & 3 & 4 & 3 & 5 & 10 & 6 & 1 \\
\hline $\mathrm{r}_{-} 15 \mathrm{~b} \_8$ & 6 & 3 & 3 & 4 & 3 & 5 & 10 & 6 & 1 \\
\hline $\mathrm{r}_{-} 15 \mathrm{~b} \_7$ & 6 & 3 & 3 & 4 & 3 & 5 & 10 & 6 & 1 \\
\hline $\mathrm{r}_{-} 15 \mathrm{~b} \_6$ & 7 & 3 & 3 & 4 & 3 & 5 & 10 & 6 & 1 \\
\hline r_20 b_9 & 5 & 3 & 2 & 3 & 5 & 4 & 3 & 7 & 2 \\
\hline $\mathrm{r} \_20 \mathrm{~b} \_8$ & 5 & 3 & 2 & 3 & 5 & 4 & 3 & 7 & 2 \\
\hline r_20 b_ 7 & 5 & 3 & 2 & 3 & 5 & 4 & 3 & 7 & 2 \\
\hline r_20 b_6 & 4 & 3 & 2 & 3 & 5 & 4 & 3 & 7 & 2 \\
\hline r_5 a_10 & 8 & 5 & 6 & 6 & 4 & 4 & 9 & 5 & 2 \\
\hline r_5 a_100 & 8 & 5 & 6 & 6 & 4 & 4 & 9 & 5 & 2 \\
\hline
\end{tabular}


Table A.3 continued from previous page

\begin{tabular}{|c|c|c|c|c|c|c|c|c|c|}
\hline MRIP & picture 1 & picture 2 & picture3 & picture4 & picture5 & picture6 & picture 7 & picture 8 & picture9 \\
\hline r_5 a_1000 & 8 & 5 & 6 & 6 & 4 & 4 & 9 & 5 & 2 \\
\hline r_5 a_10000 & 8 & 4 & 11 & 7 & 5 & 7 & 23 & 15 & 5 \\
\hline $\mathrm{r}_{-} 10 \mathrm{a}_{-} 10$ & 5 & 3 & 4 & 4 & 4 & 6 & 8 & 10 & 4 \\
\hline r_10 a_100 & 5 & 3 & 3 & 4 & 4 & 6 & 8 & 10 & 4 \\
\hline$r_{-} 10$ a_1000 & 5 & 3 & 4 & 5 & 4 & 6 & 8 & 10 & 4 \\
\hline $\mathrm{r}_{-} 10 \mathrm{a}_{-} 10000$ & 5 & 3 & 4 & 5 & 4 & 6 & 8 & 10 & 4 \\
\hline $\mathrm{r}_{-} 15 \mathrm{a} \_10$ & 7 & 3 & 3 & 4 & 3 & 5 & 10 & 6 & 1 \\
\hline $\mathrm{r}_{-} 15 \mathrm{a}_{-} 100$ & 7 & 3 & 3 & 4 & 3 & 5 & 10 & 6 & 1 \\
\hline $\mathrm{r}_{-} 15$ a_$\_1000$ & 7 & 3 & 3 & 4 & 3 & 5 & 10 & 6 & 1 \\
\hline $\mathrm{r}_{-} 15 \mathrm{a}_{-} 10000$ & 6 & 3 & 3 & 4 & 3 & 5 & 10 & 6 & 1 \\
\hline $\mathrm{r}_{2} 20 \mathrm{a} \_10$ & 5 & 3 & 2 & 3 & 5 & 4 & 3 & 7 & 2 \\
\hline $\mathrm{r}_{-} 20 \mathrm{a}_{-} 100$ & 5 & 3 & 2 & 3 & 5 & 4 & 3 & 7 & 2 \\
\hline$r_{-} 20$ a_1 1000 & 5 & 3 & 2 & 3 & 5 & 4 & 3 & 7 & 2 \\
\hline r_20 a_10000 & 5 & 3 & 2 & 3 & 5 & 4 & 3 & 7 & 2 \\
\hline b_9 a_10 & 6 & 3 & 4 & 6 & 4 & 7 & 11 & 6 & 2 \\
\hline
\end{tabular}


Table A.3 continued from previous page

\begin{tabular}{|c|c|c|c|c|c|c|c|c|c|}
\hline MRIP & picture 1 & picture 2 & picture 3 & picture4 & picture 5 & picture6 & picture7 & picture 8 & picture9 \\
\hline b_9 a_100 & 6 & 3 & 4 & 6 & 4 & 7 & 11 & 6 & 2 \\
\hline b_9 a_1000 & 6 & 3 & 4 & 6 & 4 & 7 & 11 & 6 & 2 \\
\hline b_9 a_10000 & 6 & 3 & 4 & 6 & 4 & 7 & 11 & 6 & 2 \\
\hline b_8 a_10 & 6 & 3 & 4 & 6 & 4 & 7 & 11 & 6 & 2 \\
\hline b_8 a_100 & 6 & 3 & 4 & 6 & 4 & 7 & 11 & 6 & 2 \\
\hline b_8 a_1000 & 6 & 3 & 4 & 6 & 4 & 7 & 11 & 6 & 2 \\
\hline b_8 a_10000 & 6 & 3 & 4 & 6 & 4 & 7 & 12 & 6 & 2 \\
\hline $\mathrm{b}_{-} 7 \mathrm{a} \_10$ & 6 & 3 & 4 & 6 & 4 & 7 & 11 & 6 & 2 \\
\hline b_7 a_100 & 6 & 3 & 4 & 6 & 4 & 7 & 11 & 6 & 2 \\
\hline b_7 a_1000 & 6 & 3 & 4 & 6 & 4 & 7 & 11 & 6 & 2 \\
\hline $\mathrm{b}_{-} 7 \mathrm{a} \_10000$ & 6 & 3 & 4 & 6 & 4 & 7 & 11 & 6 & 2 \\
\hline b_6 a $\_10$ & 6 & 3 & 4 & 6 & 4 & 7 & 11 & 6 & 2 \\
\hline b_6 a_100 & 6 & 3 & 4 & 6 & 4 & 7 & 11 & 6 & 2 \\
\hline b_6 a_1000 & 6 & 3 & 4 & 6 & 4 & 7 & 11 & 6 & 2 \\
\hline b_6 a_10000 & 6 & 3 & 4 & 6 & 4 & 7 & 11 & 6 & 2 \\
\hline
\end{tabular}


Table A.3 continued from previous page

\begin{tabular}{|c|c|c|c|c|c|c|c|c|c|}
\hline MRIP & picture 1 & picture2 & picture 3 & picture4 & picture 5 & picture6 & picture 7 & picture 8 & picture9 \\
\hline b_9 r_5 a_10 & 7 & 5 & 6 & 6 & 4 & 4 & 9 & 5 & 2 \\
\hline b_9 r_5 a_100 & 7 & 5 & 6 & 6 & 4 & 4 & 9 & 5 & 2 \\
\hline b_9 r_5 a_1000 & 7 & 5 & 6 & 6 & 4 & 4 & 9 & 5 & 2 \\
\hline b_9 r_5 a_10000 & 7 & 5 & 6 & 6 & 4 & 4 & 9 & 5 & 2 \\
\hline b_9 r_10 a_10 & 5 & 3 & 4 & 4 & 4 & 6 & 8 & 10 & 4 \\
\hline b_9 r_10 a_100 & 5 & 3 & 4 & 4 & 4 & 6 & 8 & 10 & 4 \\
\hline b_9 $\mathrm{r}_{-} 10 \mathrm{a} \_1000$ & 5 & 3 & 3 & 5 & 4 & 6 & 8 & 10 & 4 \\
\hline b_9 r_10 a_10000 & 5 & 3 & 3 & 5 & 4 & 6 & 8 & 10 & 4 \\
\hline b_9 r_15 a_10 & 6 & 3 & 3 & 4 & 3 & 5 & 10 & 6 & 1 \\
\hline b_9 r_15 a_100 & 6 & 3 & 3 & 4 & 3 & 5 & 10 & 6 & 1 \\
\hline b_9 $\mathrm{r}_{-} 15$ a $\_1000$ & 6 & 3 & 3 & 4 & 3 & 5 & 10 & 6 & 1 \\
\hline b_9 r_15 a_10000 & 2 & 2 & 1 & 2 & 4 & 2 & 4 & 2 & 0 \\
\hline b_9 r_20 a_10 & 5 & 3 & 2 & 3 & 5 & 4 & 3 & 7 & 2 \\
\hline b_9 r 20 a_100 & 5 & 3 & 2 & 3 & 5 & 4 & 3 & 7 & 2 \\
\hline b_9 r_20 a_1000 & 5 & 3 & 2 & 3 & 5 & 4 & 3 & 7 & 2 \\
\hline
\end{tabular}


Table A.3 continued from previous page

\begin{tabular}{|c|c|c|c|c|c|c|c|c|c|}
\hline MRIP & picture 1 & picture 2 & picture 3 & picture 4 & picture 5 & picture6 & picture7 & picture 8 & picture9 \\
\hline b_9 r_20 a_10000 & 5 & 3 & 2 & 3 & 5 & 4 & 3 & 7 & 2 \\
\hline b_8 r_5 a_10 & 8 & 5 & 6 & 6 & 4 & 4 & 9 & 5 & 2 \\
\hline b_8 r_5 a_100 & 8 & 5 & 6 & 6 & 4 & 4 & 9 & 5 & 2 \\
\hline b_8 r_5 a_1000 & 8 & 5 & 6 & 6 & 4 & 4 & 9 & 5 & 2 \\
\hline b_8 r_5 a_10000 & 8 & 5 & 6 & 6 & 4 & 4 & 9 & 5 & 2 \\
\hline b_8 r_10 a_10 & 5 & 3 & 4 & 4 & 4 & 6 & 8 & 10 & 4 \\
\hline b_8 $r \_10$ a_100 & 5 & 3 & 4 & 4 & 4 & 6 & 8 & 10 & 4 \\
\hline b_8 r_10 a_1000 & 5 & 3 & 4 & 5 & 4 & 6 & 8 & 10 & 4 \\
\hline b_8 r_10 a_10000 & 5 & 3 & 3 & 5 & 4 & 6 & 8 & 10 & 4 \\
\hline b_8 $\mathrm{r}_{-} 15$ a $\_10$ & 6 & 3 & 3 & 4 & 3 & 5 & 10 & 6 & 1 \\
\hline b_8 $\mathrm{r}_{-} 15$ a $\_100$ & 6 & 3 & 3 & 4 & 3 & 5 & 10 & 6 & 1 \\
\hline b_8 r_15 a_1000 & 6 & 3 & 3 & 4 & 3 & 5 & 10 & 6 & 1 \\
\hline b_8 r_15 a_10000 & 6 & 3 & 3 & 4 & 3 & 5 & 10 & 6 & 1 \\
\hline b_8 r_20 a_10 & 5 & 3 & 2 & 3 & 5 & 4 & 3 & 7 & 2 \\
\hline b_8 r_20 a $\_100$ & 5 & 3 & 2 & 3 & 5 & 4 & 3 & 7 & 2 \\
\hline
\end{tabular}


Table A.3 continued from previous page

\begin{tabular}{|c|c|c|c|c|c|c|c|c|c|}
\hline MRIP & picture 1 & picture 2 & picture 3 & picture4 & picture 5 & picture6 & picture7 & picture8 & picture9 \\
\hline b_8 r_20 a_1000 & 5 & 3 & 2 & 3 & 5 & 4 & 3 & 7 & 2 \\
\hline b_8 r_20 a_10000 & 5 & 3 & 2 & 3 & 5 & 4 & 3 & 7 & 2 \\
\hline$b_{-}$-7 r_5 a_10 & 6 & 5 & 6 & 6 & 4 & 4 & 9 & 5 & 2 \\
\hline b_7 r_5 a_100 & 6 & 5 & 6 & 6 & 4 & 4 & 9 & 5 & 2 \\
\hline b_7 r_5 a_1000 & 6 & 5 & 6 & 6 & 4 & 4 & 9 & 5 & 2 \\
\hline b_7 r_5 a_10000 & 6 & 5 & 6 & 6 & 4 & 4 & 9 & 5 & 2 \\
\hline b_7 r_10 a_10 & 5 & 3 & 4 & 4 & 4 & 6 & 8 & 10 & 4 \\
\hline $\mathrm{b}_{-} 7 \mathrm{r}_{-} 10 \mathrm{a}_{-} 100$ & 5 & 3 & 4 & 4 & 4 & 6 & 8 & 10 & 4 \\
\hline $\mathrm{b}_{-} 7 \mathrm{r} \_10 \mathrm{a} \_1000$ & 5 & 3 & 4 & 5 & 4 & 6 & 8 & 10 & 4 \\
\hline $\mathrm{b}_{-} 7 \mathrm{r} \_10 \mathrm{a} \_10000$ & 5 & 3 & 3 & 5 & 4 & 5 & 8 & 10 & 4 \\
\hline $\mathrm{b}_{-} 7 \mathrm{r} \_15 \mathrm{a} \_10$ & 6 & 3 & 3 & 4 & 3 & 5 & 10 & 6 & 1 \\
\hline $\mathrm{b}_{-} 7 \mathrm{r} \_15 \mathrm{a}_{-} 100$ & 6 & 3 & 3 & 4 & 3 & 5 & 10 & 6 & 1 \\
\hline b_7 r_15 a_1000 & 6 & 3 & 3 & 4 & 3 & 5 & 10 & 6 & 1 \\
\hline $\mathrm{b}_{-} 7 \mathrm{r} \_15 \mathrm{a} \_10000$ & 6 & 3 & 3 & 4 & 3 & 5 & 10 & 6 & 1 \\
\hline $\mathrm{b}_{-} 7 \mathrm{r} \_20 \mathrm{a}_{-} 10$ & 5 & 3 & 2 & 3 & 5 & 4 & 3 & 7 & 2 \\
\hline
\end{tabular}


Table A.3 continued from previous page

\begin{tabular}{|c|c|c|c|c|c|c|c|c|c|}
\hline MRIP & picture 1 & picture 2 & picture 3 & picture4 & picture 5 & picture6 & picture7 & picture8 & picture9 \\
\hline $\mathrm{b}_{-} 7 \mathrm{r} \_20 \mathrm{a}_{-} 100$ & 5 & 3 & 2 & 3 & 5 & 4 & 3 & 7 & 2 \\
\hline b_7 r_20 a_1000 & 5 & 3 & 2 & 3 & 5 & 4 & 3 & 7 & 2 \\
\hline b_7 r_20 a_10000 & 5 & 3 & 2 & 3 & 5 & 4 & 3 & 7 & 2 \\
\hline b_6 r_5 a_10 & 6 & 5 & 6 & 6 & 4 & 4 & 9 & 5 & 2 \\
\hline b_6 r_5 a_100 & 6 & 5 & 6 & 6 & 4 & 4 & 9 & 5 & 2 \\
\hline b_6 r_5 a_1000 & 6 & 5 & 6 & 6 & 4 & 4 & 9 & 5 & 2 \\
\hline b_6 r_5 a_10000 & 6 & 5 & 5 & 6 & 4 & 4 & 9 & 5 & 2 \\
\hline b_6 r $\_10$ a $\_10$ & 6 & 3 & 4 & 4 & 4 & 6 & 8 & 10 & 4 \\
\hline b_6 r $\_10$ a $\_100$ & 6 & 3 & 4 & 4 & 4 & 6 & 8 & 10 & 4 \\
\hline b_6 r_10 a_1000 & 6 & 3 & 4 & 5 & 4 & 6 & 8 & 10 & 4 \\
\hline b_6 r_10 a _ 10000 & 6 & 3 & 3 & 4 & 4 & 6 & 8 & 10 & 4 \\
\hline b_6 r__15 a_10 & 7 & 3 & 3 & 4 & 3 & 5 & 10 & 6 & 1 \\
\hline b_6 r $\_15$ a_100 & 7 & 3 & 3 & 4 & 3 & 5 & 10 & 6 & 1 \\
\hline b_6 r_15 a_1000 & 7 & 3 & 3 & 4 & 3 & 5 & 10 & 6 & 1 \\
\hline b_6 r_15 a_10000 & 7 & 3 & 3 & 4 & 3 & 5 & 10 & 6 & 1 \\
\hline
\end{tabular}


Table A.3 continued from previous page

\begin{tabular}{|c|c|c|c|c|c|c|c|c|c|}
\hline MRIP & picture 1 & picture 2 & picture 3 & picture4 & picture 5 & picture6 & picture 7 & picture8 & picture9 \\
\hline b_6 r_20 a_10 & 4 & 3 & 2 & 3 & 5 & 4 & 3 & 7 & 2 \\
\hline b_6 r_20 a_100 & 4 & 3 & 2 & 3 & 5 & 4 & 3 & 7 & 2 \\
\hline b_6 r_20 a_1000 & 4 & 3 & 2 & 3 & 5 & 4 & 3 & 7 & 2 \\
\hline b_6 r_20 a_10000 & 4 & 3 & 2 & 3 & 5 & 4 & 3 & 7 & 2 \\
\hline
\end{tabular}




\section{Appendix B}

\section{B.1 The execution time of the experiment}

Table B.1: The execution time records of the experiment

\begin{tabular}{|c|c|c|}
\hline MRIP combination & execution time & average time \\
\hline rotate 5 & 14.56 & \multirow{4}{*}{14.54} \\
\hline rotate 10 & 14.3 & \\
\hline rotate 15 & 14.53 & \\
\hline rotate 20 & 14.76 & \\
\hline brightness 0.9 & 1.07 & \multirow{4}{*}{1.06} \\
\hline brightness 0.8 & 1.05 & \\
\hline brightness 0.7 & 1.05 & \\
\hline brightness 0.6 & 1.06 & \\
\hline noise 10 & 0.68 & \multirow{4}{*}{0.66} \\
\hline noise 100 & 0.65 & \\
\hline noise 1000 & 0.67 & \\
\hline noise 10000 & 0.65 & \\
\hline rotate 5 brightness 0.9 & 14.97 & \\
\hline rotate 5 brightness 0.8 & 14.79 & \\
\hline rotate 5 brightness 0.7 & 14.95 & \\
\hline rotate 10 brightness 0.6 & 14.68 & \\
\hline
\end{tabular}


Table B.1 continued from previous page

\begin{tabular}{|c|c|c|}
\hline MRIP combination & execution time & average time \\
\hline rotate 10 brightness 0.9 & 14.56 & \\
\hline rotate 10 brightness 0.8 & 15.01 & \\
\hline rotate 10 brightness 0.7 & 14.91 & \\
\hline rotate 10 brightness 0.6 & 14.67 & \\
\hline rotate 15 brightness 0.9 & 15.04 & \\
\hline rotate 15 brightness 0.8 & 14.56 & \\
\hline rotate 15 brightness 0.7 & 14.65 & \\
\hline rotate 15 brightness 0.6 & 14.77 & \\
\hline rotate 20 brightness 0.9 & 14.58 & \\
\hline rotate 20 brightness 0.8 & 14.98 & \\
\hline rotate 20 brightness 0.7 & 14.95 & \\
\hline rotate 20 brightness 0.6 & 14.85 & \\
\hline rotate 5 noise 10 & 14.98 & \multirow{15}{*}{14.98} \\
\hline rotate 5 noise 100 & 15.23 & \\
\hline rotate 5 noise 1000 & 14.87 & \\
\hline rotate 5 noise 10000 & 15.24 & \\
\hline rotate 10 noise 10 & 15.01 & \\
\hline rotate 10 noise 100 & 15.16 & \\
\hline rotate 10 noise 1000 & 14.88 & \\
\hline rotate 10 noise 10000 & 14.99 & \\
\hline rotate 15 noise 10 & 15.12 & \\
\hline rotate 15 noise 100 & 14.52 & \\
\hline rotate 15 noise 1000 & 14.58 & \\
\hline rotate 15 noise 10000 & 15.17 & \\
\hline rotate 20 noise 10 & 15.06 & \\
\hline rotate 20 noise 100 & 15.08 & \\
\hline rotate 20 noise 1000 & 14.92 & \\
\hline
\end{tabular}


Table B.1 continued from previous page

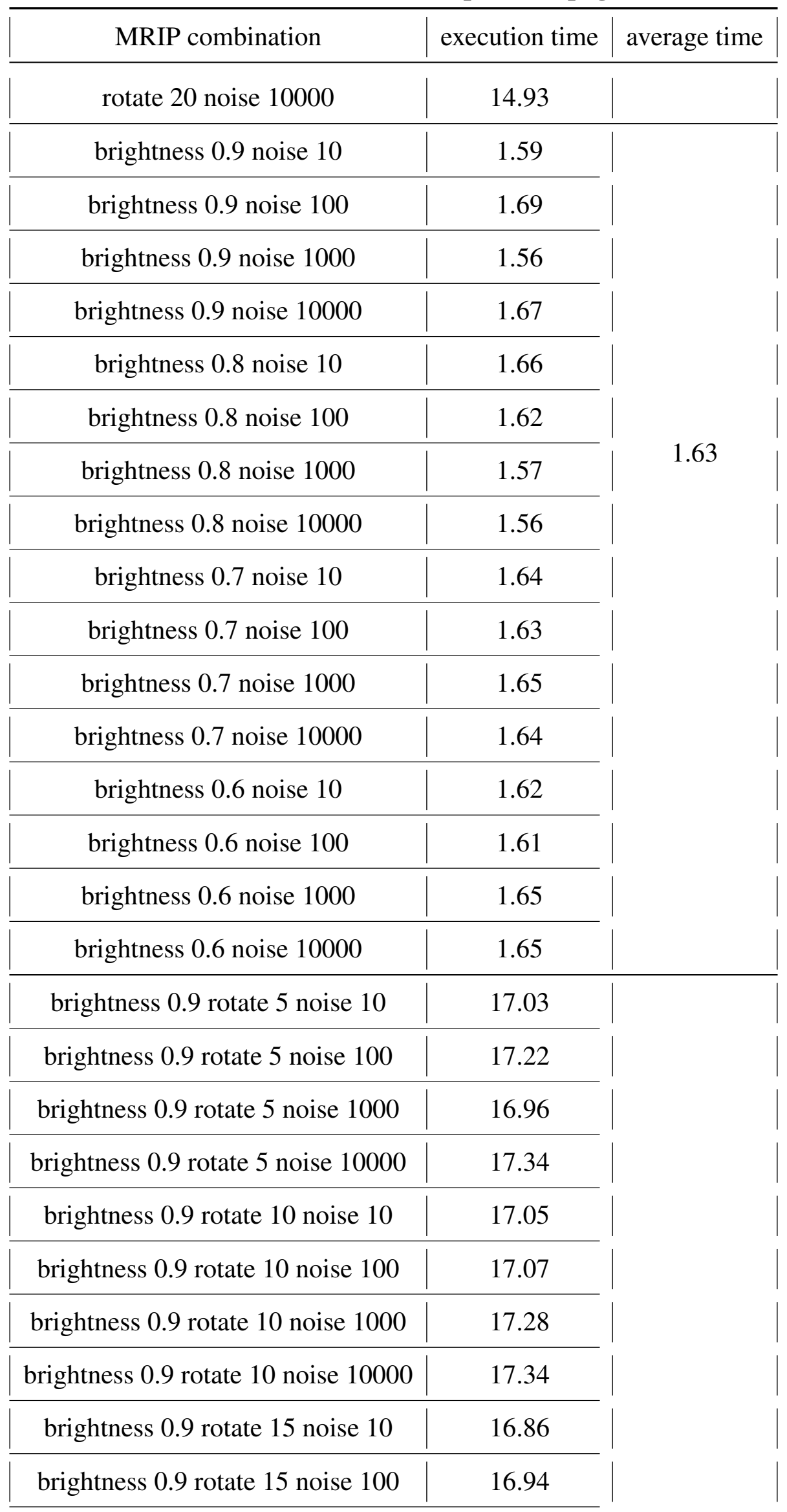


Table B.1 continued from previous page

\begin{tabular}{|c|c|c|}
\hline MRIP combination & execution time & average time \\
\hline brightness 0.9 rotate 15 noise 1000 & 16.36 & \\
\hline brightness 0.9 rotate 15 noise 10000 & 16.96 & \\
\hline brightness 0.9 rotate 20 noise 10 & 17.96 & \\
\hline brightness 0.9 rotate 20 noise 100 & 16.46 & \\
\hline brightness 0.9 rotate 20 noise 1000 & 16.45 & \\
\hline brightness 0.9 rotate 20 noise 10000 & 16.95 & \\
\hline brightness 0.8 rotate 5 noise 10 & 16.86 & \\
\hline brightness 0.8 rotate 5 noise 100 & 17.31 & \\
\hline brightness 0.8 rotate 5 noise 1000 & 17.34 & \\
\hline brightness 0.8 rotate 5 noise 10000 & 17.01 & \\
\hline brightness 0.8 rotate 10 noise 10 & 17.05 & \\
\hline brightness 0.8 rotate 10 noise 100 & 17.04 & \\
\hline brightness 0.8 rotate 10 noise 1000 & 17.05 & \\
\hline brightness 0.8 rotate 10 noise 10000 & 17.32 & \\
\hline brightness 0.8 rotate 15 noise 10 & 17.21 & \\
\hline brightness 0.8 rotate 15 noise 100 & 17.05 & \\
\hline brightness 0.8 rotate 15 noise 1000 & 17.34 & \\
\hline brightness 0.8 rotate 15 noise 10000 & 16.33 & \\
\hline brightness 0.8 rotate 20 noise 10 & 17.32 & \\
\hline brightness 0.8 rotate 20 noise 100 & 17.86 & \\
\hline brightness 0.8 rotate 20 noise 1000 & 17.33 & \\
\hline brightness 0.8 rotate 20 noise 10000 & 17.32 & \\
\hline brightness 0.7 rotate 5 noise 10 & 17.36 & \\
\hline brightness 0.7 rotate 5 noise 100 & 17.39 & \\
\hline brightness 0.7 rotate 5 noise 1000 & 17.35 & \\
\hline brightness 0.7 rotate 5 noise 10000 & 17.65 & \\
\hline brightness 0.7 rotate 10 noise 10 & 17.72 & \\
\hline
\end{tabular}


Table B.1 continued from previous page

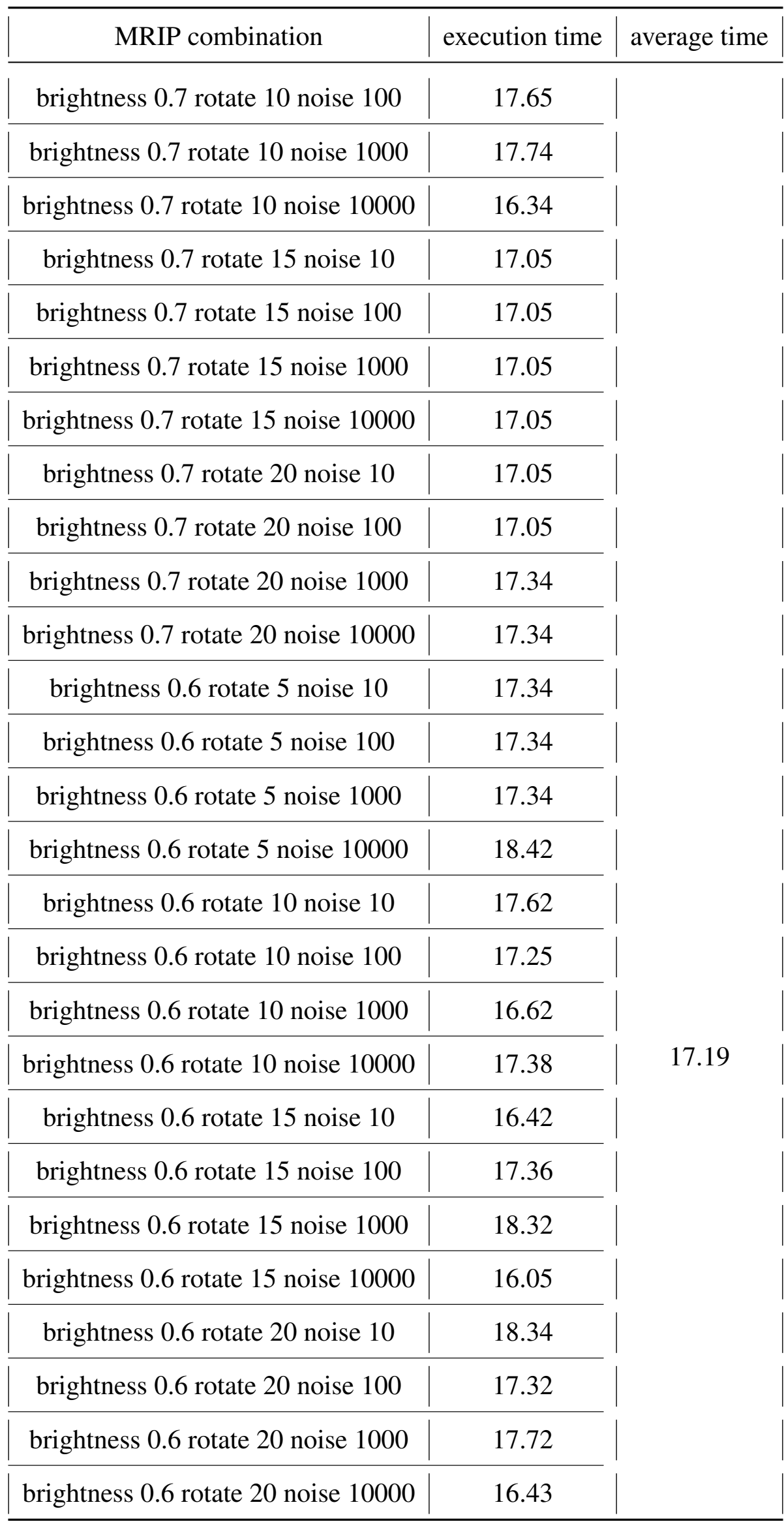




\section{Appendix C}

\section{C.1 Documentation of MTKeras}

\section{C.1.1 MTKeras documentation}

MTKeras is a fully-automated platform that facilitates MT for users (both industrial and academic) who may not have much experience in metamorphic testing.

\section{C.1.2 Authors}

Yelin Liu and Zhi Quan Zhou, University of Wollongong and Morphick Solutions Pty Ltd, Australia.

\section{C.1.3 Table of Contents}

\section{Overview}

APIs

Examples

\section{Glossary}

\section{C.1.4 Overview}

Machine learning (ML) platforms and libraries, such as TensorFlow and Theano, are now widely available to allow users to develop and train their own ML models. We have built our MT framework, MTKeras, on the Keras platform. Keras (https://keras.io) is a popular high-level neural networks API, developed in Python and working on top of low-level libraries-those backend engines such as Tensorflow and Theano can be plugged seamlessly into Keras. The Keras API empowers users to configure and train a neural network 
model based on datasets for various tasks such as image classification or sentiment analysis. MTKeras enables automated metamorphic testing by providing the users with an MR library for testing their ML models and applications. We have designed the MR library based on the concept of a hierarchical structure (levels of abstractions) of MRPs. MTKeras also allows the users to define and run new MRs through the composition of multiple MRs. The source test cases are provided by the users whereas followup test cases are generated by MTKeras. MR violation tests are automatically recorded during testing.

\section{C.1.5 APIs}

\section{Implementation:}

- 1. Download the Mtkeras package to the same folder of the targeted script (the Mtkeras package should be in the same folder of the target script). Otherwise, the user can download the package from github(https://github.com/lawrence415610/Mtkeras.git)

- 2. Import the class using the command:

from Mtkeras.Mtkeras import Mtkeras

- 3. The user can perform MT in a simple and intuitive way by writing a single line of code in the following format:

$$
\operatorname{Mtkeras}(<\text { sourceTestSet }>,<\text { dataType }>) .<\text { MRIPs }>[.<\text { MROP }>]
$$

\section{Args:}

- myTestSet: an array or ndarray that contains image data or other kind of data. Each pieces of data should be an array, and all these array should be stored in one array, which is the myTestSet array.

- dataType: a string that can represent the context of the SUT, it can be: "grayscaleImage", "colorImage" and "text"

- model: an object. It is the neural network model under test, if the Mtkeras is only used for test case generation, this argument can be omitted. The "model" argument is needed only when MROP is performed.

\section{Returns:}

It will return a Mtkeras object, by calling different attributes, the returns will be different.

- return a transformed dataset(an array/ndarray), call the attribute ".myTestSet" 
- return a dataset of violating cases, call the attribute ".violatingCases"

\section{permutative:}

- Summary: The "permutative" MRIP: the user can shuffle the order of the data randomly in the dataset

- Args: None

- Returns:

1. a Mtkeras Object

2. call the property ".myTestSet" to return a transformed dataset(an array/ndarray)

\section{additive:}

- Summary: The "additive" MRIP: increase (or decrease) numerical values by a constant for each pieces of data in the dataset

- Args: - $\mathrm{n} \_$additive: integer, the constant that is used to change each pieces of data

- Returns:

1. a Mtkeras Object

2. call the property ".myTestSet" to return a transformed dataset(an array/ndarray)

\section{brightness:}

- Summary: The "brightness" MRIP: for images, this MRIP can adjust the image's brightness. It transforms the input image pixel-wise according to the equation $\mathrm{O}=$ $I^{* *}$ gamma after scaling each pixel to the range 0 to 1

- Args:

1. gamma(optional): float, non negative real number, the default value is 1

2. gain(optional): float, the constant multiplier. Default value is 1

- Returns:

1. a Mtkeras Object 2. call the property ".myTestSet" to return a transformed dataset(an array/ndarray)

\section{multiplicative:}

- Summary: the "multiplicative" MRIP: multiply numerical values by a constant for each pieces of data in the dataset

- Args: $\mathrm{n}$ mul: integer, the constant used for multiplying the dataset 
- Returns:

1. an Mtkeras Object

2. call the property ".myTestSet" to return a tranformed dataset(an array/ndarray)

\section{invertive:}

- Summary: the "invertive" MRIP: invert the element in the dataset

- Args: None

- Returns:

1. an Mtkeras Object

2. call the property ".myTestSet" to return a tranformed dataset(an array/ndarray)

\section{noise:}

- Summary: the "noise" MRIP: create one or more noise points in a dataset

- Args: $\mathrm{n} \_$noise: integer, $\mathrm{n} \_$noise $i=0$, the number of the noise point that is added to the dataset

- Returns:

1. an Mtkeras Object 2. call the property ".myTestSet" to return a transformed dataset(an array/ndarray)

\section{fliph:}

- Summary: the "fliph" MRIP: flip the data horizontally

- Args: None

- Returns:

1. an Mtkeras Object 2. call the property ".myTestSet" to return a transformed dataset(an array/ndarray)

\section{flipv:}

- Summary: the "flipv" MRIP: flip the data vertically

- Args: None

- Returns:

1. an Mtkeras Object 2. call the property ".myTestSet" to return a transformed dataset(an array/ndarray) 


\section{rotate:}

- Summary: the "rotation" MRIP: rotate the image

- Args: -n_deg: float, specify the degree that the image rotate

- Returns:

1. an Mtkeras Object 2. call the property ".myTestSet" to return a transformed dataset(an array/ndarray)

\section{NoREC:}

- Summary: the "NoREC" MRIP: When an SQL query is rewritten as an optimized version, the number of results returned by the DBMS should remain the same.

- Args: 1. mySQL

- Returns:

1. an Mtkeras Object

2. call the property ".myTestSet" to return an optimized SQL

equal:

- Summary: the "equal" MROP: the output of the first dataset should be equal to the second test dataset

- Args: none

- Returns:

1. an Mtkeras Object

2. call the property ".myTestSet" to return a transformed dataset(an array/ndarray)

3 . call the property ".violatingCases" to return a dataset of violating cases

- Outputs:

the number of the violation cases will be printed

\section{subset:}

- Summary: the "subset" MROP: the output of the first dataset should be subset to the second test dataset

- Args: none

- Returns:

1. an Mtkeras Object

2. call the property ".violatingCases" to return a dataset of violating cases 
- Outputs:

the number of the violation cases will be printed

\section{disjoint:}

- Summary: the "disjoint" MROP: the output of the first dataset should be disjoint to the second test dataset

- Args: none

- Returns:

1. an Mtkeras Object

2. call the property ".violatingCases" to return a dataset of violating cases

- Outputs:

the number of the violation cases will be printed

\section{complete:}

- Summary: the "complete" MROP: the output of the first dataset should be disjoint to the second test dataset

- Args: none

- Returns:

1. an Mtkeras Object

2. call the property ".violatingCases" to return a dataset of violating cases

- Outputs:

the number of the violation cases will be printed

\section{difference:}

- Summary: the "difference" MROP: the output of the first dataset should be different to the second test dataset

- Args: none

- Returns:

1. an Mtkeras Object

2. call the property ".violatingCases" to return a dataset of violating cases

- Outputs:

the number of the violation cases will be printed 


\section{C.1.6 License}

MIT License

Copyright (c) 2020 Yelin Liu, Zhiquan (George) Zhou

Permission is hereby granted, free of charge, to any person obtaining a copy of this software and associated documentation files (the "Software"), to deal in the Software without restriction, including without limitation the rights to use, copy, modify, merge, publish, distribute, sublicense, and/or sell copies of the Software, and to permit persons to whom the Software is furnished to do so, subject to the following conditions:

The above copyright notice and this permission notice shall be included in all copies or substantial portions of the Software.

THE SOFTWARE IS PROVIDED "AS IS", WITHOUT WARRANTY OF ANY KIND, EXPRESS OR IMPLIED, INCLUDING BUT NOT LIMITED TO THE WARRANTIES OF MERCHANTABILITY, FITNESS FOR A PARTICULAR PURPOSE AND NONINFRINGEMENT. IN NO EVENT SHALL THE AUTHORS OR COPYRIGHT HOLDERS BE LIABLE FOR ANY CLAIM, DAMAGES OR OTHER LIABILITY, WHETHER IN AN ACTION OF CONTRACT, TORT OR OTHERWISE, ARISING FROM, OUT OF OR IN CONNECTION WITH THE SOFTWARE OR THE USE OR OTHER DEALINGS IN THE SOFTWARE.

\section{C.1.7 Glossary}

- Machine Learning testing(ML testing): activities designed to reveal the flaws in machine learning items that can result in discordance between the existing and the required conditions.

- Test Oracle: A test oracle is a mechanism that can verify the test case results.

- Metamorphic Testing(MT): a testing technique to alleviate the test oracle problem.

- Metamorphic Relation(MR): the relations between inputs and outputs are defined as metamorphic relations which is considered as "derived oracles" that can alleviate the test oracle problem.

- Metamorphic Relation Pattern(MRP): defined the abstraction that features a set of (possibly infinitely many) metamorphic relations.

- Metamorphic Relation Input Pattern (MRIP): an abstraction that characterizes the relations among the source and follow-up inputs of a set of (possibly infinitely many) metamorphic relations. 
- Metamorphic Relation Output Pattern(MROP): an abstraction that characterizes the relations among the source and follow-up outputs of a set of (possibly infinitely many) metamorphic relations. 
Appendix D

D.1 Survey Design 


\section{MTKeras Usability Survey \\ datareachable Pty Ltd}

1. How comprehensive the concept of Metamorphic testing is?

A great deal

A lot

A moderate amount

A little

None at all

2. How comprehensive the concept of Metamorphic Relation Pattern is?

A great deal

A lot

A moderate amount

A little

None at all

3. How efficiently is MTKeras experiment conducted?

Extremely efficiently

$\bigcirc$ Very efficiently

Somewhat efficiently 
Not so efficiently
Not at all efficiently

4. How likely is it that you would recommend MTKeras to a friend or colleague?

Not at all likely Extremely likely

\begin{tabular}{|l|l|l|l|l|l|l|l|l|l|l|}
\hline 0 & 1 & 2 & 3 & 4 & 5 & 6 & 7 & 8 & 9 & 10 \\
\hline
\end{tabular}

\title{
Done
}

\author{
Powered by \\ क. S. SurveyMonkey \\ See how easy it is to create a survey.
}

Privacy \& Cookie Notice 